\title{
Loosely Coupled Heterogeneous Networks Convergence using IMS-SIP-AAA
}

\author{
G. Vijayalakshmy \\ Perunthalaivar Kamarajar \\ Institute of Engineering and \\ Technology \\ Pondicherry University \\ India
}

\author{
G. Sivaradje \\ Pondicherry Engineering \\ College \\ Pondicherry University \\ India
}

\begin{abstract}
In this paper a heterogeneous network model based on the IMS-SIP (session Initiation protocol) with the help of Authentication, Authorization, and Accounting (AAA) protocol that integrates the Worldwide Interoperability for Microwave Access (WiMAX), Universal Mobile Telecommunications System (UMTS) and Wireless Local Area Network (WLAN) technologies is proposed to improve QoS. The proposed Loose coupled WiMAX-UMTS-WLAN convergence architecture using IMS is compared with interworking of WiMAX-UMTS-WLAN without IMS and WiMAX-UMTS interworking with IMS. The QoS performance metrics such as different traffic delays such as Voice over IP (delay sensitive), Video streaming, Web browsing and background traffic (email, FTP), throughput are analyzed and simulated using OPNET 14.5.
\end{abstract}

\section{Keywords}

WiMAX, UMTS, WLAN, Interworking, QoS, IMS, SIP, Loose, Convergence, Heterogeneous

\section{INTRODUCTION}

Nowadays, several different wireless and wired network technologies exist that try to satisfy the different user needs and requirements. In the near future, network operators will try to combine various technologies in order to build large heterogeneous networks in order to provide the users with better services. However, the design and the development of such networks requires the consideration of different issues, such as the session control, the authorization, the authentication, the Quality of Service (QoS), the charging, the users' mobility, etc. IMS-based network architecture for WiMAX-UMTS and WiMAX-WLAN interworking is discussed in [1].The IP Multimedia Subsystem (IMS) [2] comes as a promising solution to the above issues, since it offers the needed interworking environment for the integration of, in principle, any broadband wireless access technology whilst complemented with the necessary gateways it provides access to legacy telecommunications switching systems. Also, since the IMS uses the Session Initiation Protocol (SIP) [3] for session establishment, management, and transformation, its offerings include functions, such as authentication, addressing, routing capability negotiation, service invocation, provisioning, charging, session establishment, etc. However, since the IMS networks are still in an ongoing activity in the industry and the research community constantly tries to face open issues and ex-tend IMS beyond 3G, by proposing interworking architectures that aim on seamless service provisioning. The IMS is a framework that was first designed and standardized by the 3GPP (3rd Generation Partnership Project) [2, 4] with main goal the provision of the Internet Protocol (IP) [5] based telephony and multimedia services over $3 \mathrm{G}$ networks. It has been also extended by the 3GPP, 3GPP2 and TISPAN towards integrating different broadband wireless technologies, such as WLAN, CDMA2000, UMTS, WiMAX and also fixed line networks. The IMS provides horizontally integrated services and access independence supported by functions such as authentication, addressing, routing capability negotiation, service invocation, provisioning, charging, session establishment, etc. The crucial technology for supporting multimedia session establishment and controlling information negotiation in the IMS is the SIP. Specified by the IETF, SIP supports a wide variety of applications, such as video conferencing, streaming multimedia distribution, instant messaging and presence information. A session can be modified in terms of changing addresses or port numbers, adding or deleting media streams or inviting more users to the session. The latest version of the protocol is specified in RFC 3261 [3] .Another protocol widely used in the IMS is DIAMETER, specified in RFC 3588 [7] that is used for the AAA procedure and for the exchange of some information between IMS entities. DIAMETER is an extension of RADIUS specified in RFC 2865 [8]. The main methods of Security provisioning in DIAMETER are IP Seconds as specified in RFC 2401 [9] and Transport Layer Security (TLS) specified in RFC 2246 [10]. There are also several protocols that are currently used or could possibly be used in the IMS, mainly in the application-layer, protocols used in the IMS. These include: The Common Open Policy Service (COPS) protocol specified in RFC 2748 [11] that incorporates a model for supporting policy control over QoS signaling protocols e.g. Resource Reservation Protocol (RSVP) and the transfer of policies between the Policy Decision Points and the Policy Enforcement Points. The H.248 protocol (ITU-T Recommendation H.248 [12]), also referred to as Media Gateway Control (MEGACO) protocol, that is used for controlling media gateways on the IP and PSTN networks. The Real-time Transport Protocol (RTP) specified in RFC 3550 [13] that provides support for real-time media transportation. The RTP Control Protocol (RTCP) which is always being used along with the RTP, provides QoS information and statistics whilst supports the inter-media synchronization, and The Second sure RTP (SRTP), specified in RFC 3711 [14], which provides message authentication and confidentiality to RTP/RTCP traffic. In support of IMS' endto-end QoS offerings, various link-layer resource reservation protocols could be used including the PDP (Packet Data Protocol) Context Activation, the DiffServ and the RSVP protocols. Since the IMS networks are still in an ongoing activity, researchers and operators are trying to solve open issues and extend the IMS beyond $3 \mathrm{G}$ towards to a seamless universal wireless Next Generation Network (NGN). The use of the IMS to connect different wireless Access Networks 
(ANs) including legacy networks would offer several advantages to the users and the operators. Standardization bodies and researchers have been trying to find ways to achieve this and several interworking architectures have been proposed focusing on seamless service provisioning and service continuity during handoffs. In principle, there are two main interworking methods between wireless ANs: loose coupling and tight coupling. In a loose couple configuration, the changes to the existing access technologies remain at the bare minimum; thus it guarantees the independence in terms of deployment. Alternatively, the tight coupled configuration imposes significant modifications to the protocols, interfaces and the services of the access networks. Since in this configuration the data streams pass through the 3GPP core network; the handoff latency is reduced and furthermore seamless mobility is supported. Additional interconnection levels can be found in the current research literature representing different operational capabilities. Taking into account the six interconnection levels specified by the 3GPP for WLAN-3GPP interworking [15], which in principle can be applied to any 3GPP interconnection model with other IPbased wireless access technologies, a classification of the interworking architectures is presented based on the level of coupling between the interconnected access networks and their mapping to the 3GPP interworking levels. A comparative analysis of basic and hybrid queuing methods and their impact on the VoIP traffic delay within the network is discussed in [16]. Most of the researchers address Voice analysis or video streaming analysis for integrated networks to achieve QoS. Here the motivation is to address all type of delay sensitive and non delay sensitive traffic analysis simultaneously for integrated WiMAX-UMTS-WLAN with IMS-SIP-AAA protocol. In this paper, a loosely coupled interworking model that integrates WiMAX, UMTS and WLAN in an IMS-SIP compatible architecture along with multi AAA serving protocol is proposed. Additionally IP Hybrid QoS algorithm is used to enhance the QoS performance of different types of traffic. More specifically the proposed novel architecture incorporates a UMTS, a WiMAX network and a WLAN network interconnected with the UMTS Core through specific functional entities and an IMS in charge of sessions' control. Thus, users can access the UMTS Circuit-Switched (CS) based services through the WiMAX and WLAN networks, since they are authenticated in the Authentication, Authorization, and Accounting (AAA) Server and registered in the IMS-SIP core. The rest of the paper is organized as follows. Section 2 presents about loosely coupled WiMAX-UMTS interworking architecture with IMSSIP-AAA, while Section 3 discuss about proposed Loose coupled WiMAX-UMTS-WLAN interworking architecture with IMS-SIP-AAA and without IMS. Simulation results that are used to evaluate the performance of proposed architecture are presented in Section 4. Finally, Section 5 concludes the work and discusses some future research directions.

\section{LOOSELY COUPLED WIMAX-UMTS INTERWORKING ARCHITECTURE WITH IMS-SIP-AAA}

\subsection{Loose coupling interworking}

In the loose coupling converging architecture, networks are interconnected to each other independently. The Networks are connected through the Internet Protocol (IP) backbone and indirectly connected to Core Network. Three promising wireless technologies, i.e., UMTS, WLAN and WiMAX, are integrated to form a unified network. Gateways of all networks which are to be integrated are connected through AAA server which in turn finally connects to the internet backbone [17]. Hence, there is no direct connection between the networks. Subsequently, Networks data traffic directly passes to the internet instead of core network. This approach enables combined billing, authentication, authorization and Accounting for all integrated networks through AAA server. The main advantage of using the loose coupling architecture is that it allows the deployment of independent operation of networks. This allows the different network service providers to take advantage of already existing and deployed networks. Moreover, no need to make complex enhancements; hence cost is also reduced. Subscribers can use all integrated wireless access networks while subscribing to only one service provider. For example, a WiMAX subscriber can reuse its Subscriber Identity Module (SIM) or User Service Identity Module (USIM) for UMTS or WiLAN multimedia services [17].

\subsection{Interworking of Loosely coupled WiMAX-UMTS interworking architecture} Interworking of Loosely coupled WiMAX-UMTS interworking architecture with IMS is discussed in this section. Loose coupled WiMAX-3G Interworking Architecture with IMS is shown in Figure 2. WiMAX-WAG is indirectly interconnected to $3 \mathrm{G}$ (UMTS) through IP Multimedia Subsystem through Internet backbone using AAA server. For Bulky traffic loosely coupled architecture is chosen. 


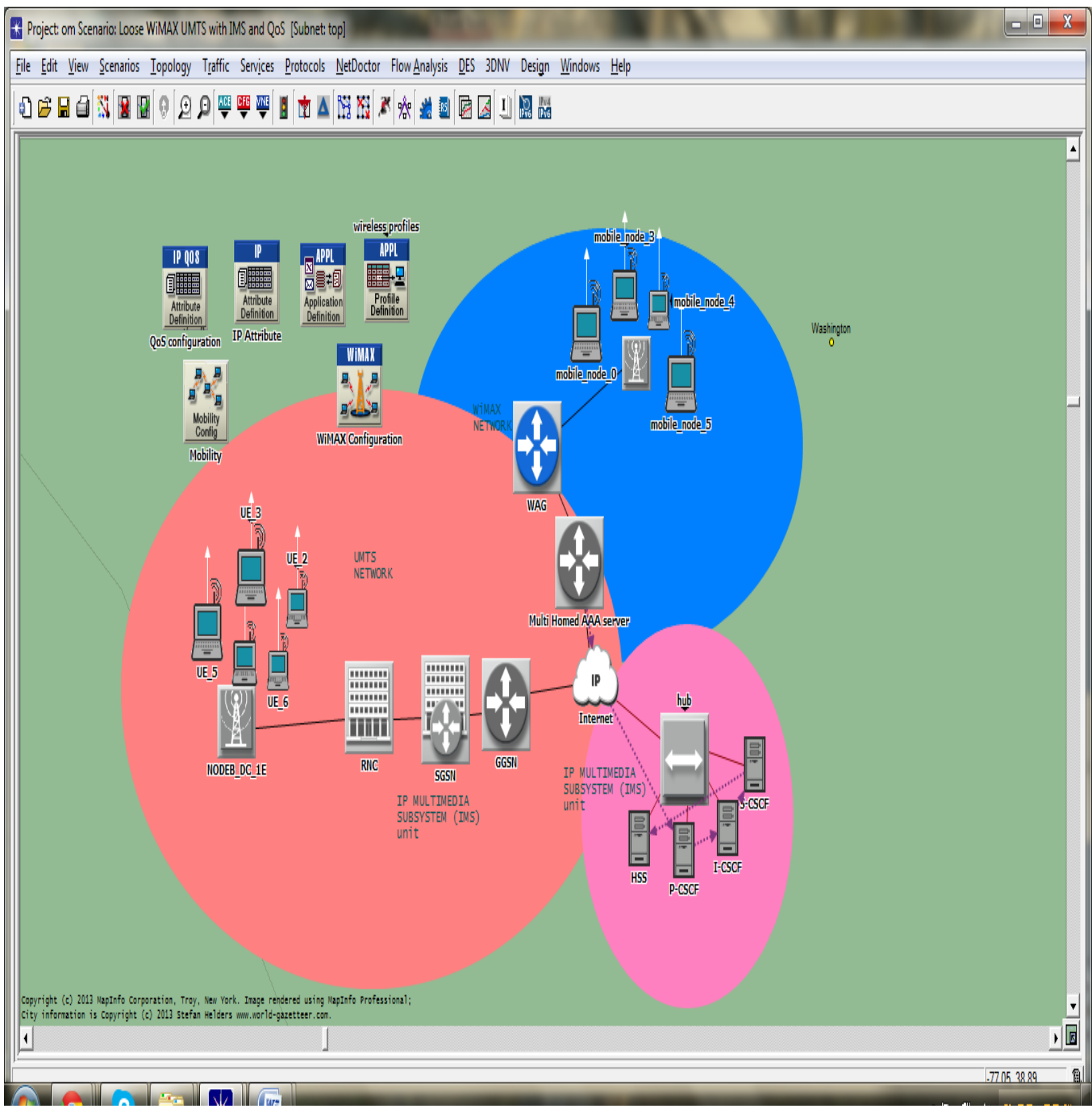

Figure 2: Loosely coupled WiMAX-UMTS Interworking Architecture with IMS

Figure 2 shows the Loose coupled WiMAX-UMTS Interworking Architecture with IMS. In this architecture QoS negotiations, Resource Reservation, Session Convergence and Session Mobility are controlled by IP multimedia subsystem where Session Initiation Protocol as the main key element. IP QoS Hybrid Queuing algorithm is configured to this Loose coupled WiMAX-3G interworking architecture for scheduling mechanism. The proposed HLLCQ (Hybrid Low latency custom Queuing) method is used to provide both priority level Queuing in addition with capabilities of Weighted Fair Queuing properties. The proposed HLLCQ algorithm is designed exclusively for converged networks that carry Background traffic, Web browsing, voice and interactive video [16]. It contains three important components, a Priority level for real time delay sensitive traffic (Voice, Video), Custom level for non delay sensitive traffic allocating the available bandwidth among all active network applications so that overload cannot appear and Weighted Fair Queuing algorithm with class based technique for fair data distribution and to avoid starving. In Proposed HLLCQ, allows delaysensitive data, such as voice, video to be sent first, before packets in other queues are de-queued and also the step traffic is managed by assigning weighted amounts, and is arranged into 16 queues. In HLLCQ, as a first step Custom level Queuing algorithm allocates the readily available resources among all active network applications so that collision may not appear. This is the main reason why it is used in the entry part of the Hybrid queuing mechanism scheme. In the second step of the algorithm Priority level is followed where the traffic is arranged into waiting queues according to the priorities set in individual packets' ToS fields. According to the ToS priorities the packets are arranged by their importance to four different internal priority queues [16]. In the third step, the output interface of the algorithm first serves the highestpriority data stream (packets that are in the queue with the highest importance) and then all other lower ranking queues. 
Then they are again scheduled into already defined classes using Class Based Weighted Fair Queuing mechanism. Such defined classes already have the needed resources prereserved as set by the network administrator. This way the packets at the final step do not need to fight for resources, as it is guaranteed in advance [16]. VoIP traffic and video traffic will already have provided a sufficient pre-allocated bandwidth, meaning that its pre-reserved bandwidth cannot be used by any other application or other traffic flow, whose classification does not fulfill the terms of class reservation. This way the output connection can transmit even the lowerpriority traffic parallel to the high-priority traffic. The proposed Hybrid Scheduling Algorithm is shown in figure $2 \mathrm{a}$

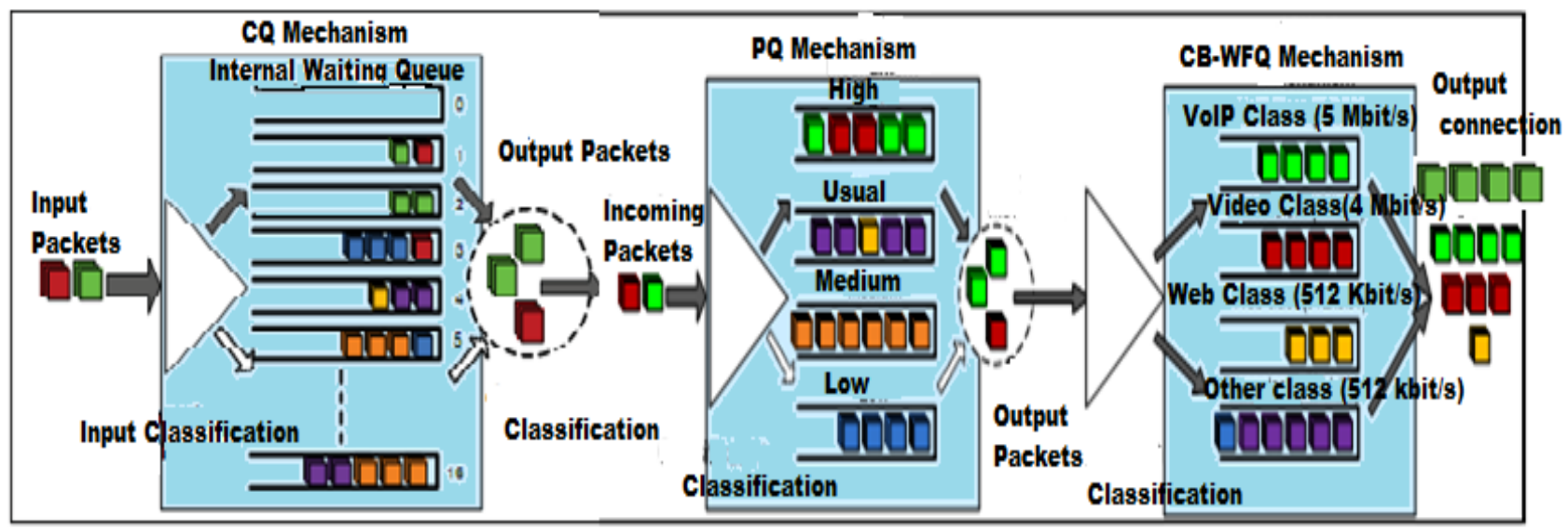

Figure 2 a: Hybrid IP QoS algorithm

In the WiMAX configuration module, mobility and ranging enable mode is selected for the better mobility support and QoS provisioning procedure. Here UMTS base station is WiMAX Base station is connected with Wireless Access Gateway (WAG) and in turn connected indirectly to UMTSGGSN to support heavy multimedia traffic through Internet backbone using AAA serving Gateway. Here AAA server is connected with UMTS GGSN and WiMAX-WAG. IMS multimedia supporting server (SIP) session initiation protocol is connected to the Ethernet hub. The HSS is the master database user profile is stored. A Home Network may contain one or several HSSs, depending on the number of mobile subscribers, on the capacity of the physical component where the HSS resides and on the overall structure and design of the network. The CSCF is an essential node in the IMS that processes the SIP signaling. There are three types of CSCFs, referred to also as SIP Servers: the Proxy-CSCF (P-CSCF), the Interrogating-CSCF (I-SCSF) and the Serving-CSCF (SCSCF) servers [1]. The P-CSCF server is the first point of contact for a User Equipment (UE) entering the IMS subsystem. Its address is discovered by the UE and its goals are to perform the registration of the UE, to compress/decompress SIP messages, to generate charging records and to enforce policies through the Policy Decision Function (PDF), whilst it can be located either in the Home Network or in the Visited one. I-CSCF server is the SIP proxy that resides at the administrative functional domain of the IMS. It queries the HSS in order to determine the location of a UE and routes UE's messages to its assigned S-CSCF [1]. ICSCF is usually located at the Home Network whilst towards enhancing the network's redundancy and scalability a number of I-CSCFs can be deployed in the same network. The SCSCF server is the entity the sessions and maintains the correlation between the UE's SIP addresses. An S-CSCF is assigned to each UE during its registration to the IMS, however each S-CSCF may serve more than one UEs, depending on the capacity of the physical component the SCSCF resides. The S-CSCF is responsible to forward the SIP messages to the appropriate AS and to enforce the service policies of the network operator [1]. Proxy-Call Session Control Function (P-CSCF), Serving-Call Session Control Function (S-CSCF) and Interrogating-Call Session Control Function (I-CSCF) is connected to the Hub. The SIP attributes are clearly discussed in proposed WiMAX-UMTS-WLAN interworking model.

\section{LOOSE COUPLED WIMAX-UMTS- WLAN INTEGRATED ARCHITECTURE WITH IMS-SIP WITH AAA}

The $4 \mathrm{G}$ and $5 \mathrm{G}$ networks will provide several benefits to the user and service providers. From the users' point of view; they will have an opportunity to select a network among all the converged networks. Therefore, the issues such as being out of coverage, Interference and limited capacity offered by with specific network will not exist. They also allow them to connect to the network seamless end to end service and also providing a large number of available resources. Therefore, the concept of "anywhere, at any time, and always connected with the best network" can be provided to the user. From network operators' point of view, such integrated wireless heterogeneous networks will provide efficient utilization of available network resources of each wireless network [17]. Furthermore, already deployed networks can be reused to provide anywhere at any time and always-on services, global coverage, low cost for the running session of mobile terminal and best services. Figure 3 a shows loosely coupled WiMAXUMTS-WLAN integrated architecture with IMS. Here also Proposed IP QoS provisioning algorithm is configured for proposed architecture along with IMS. 


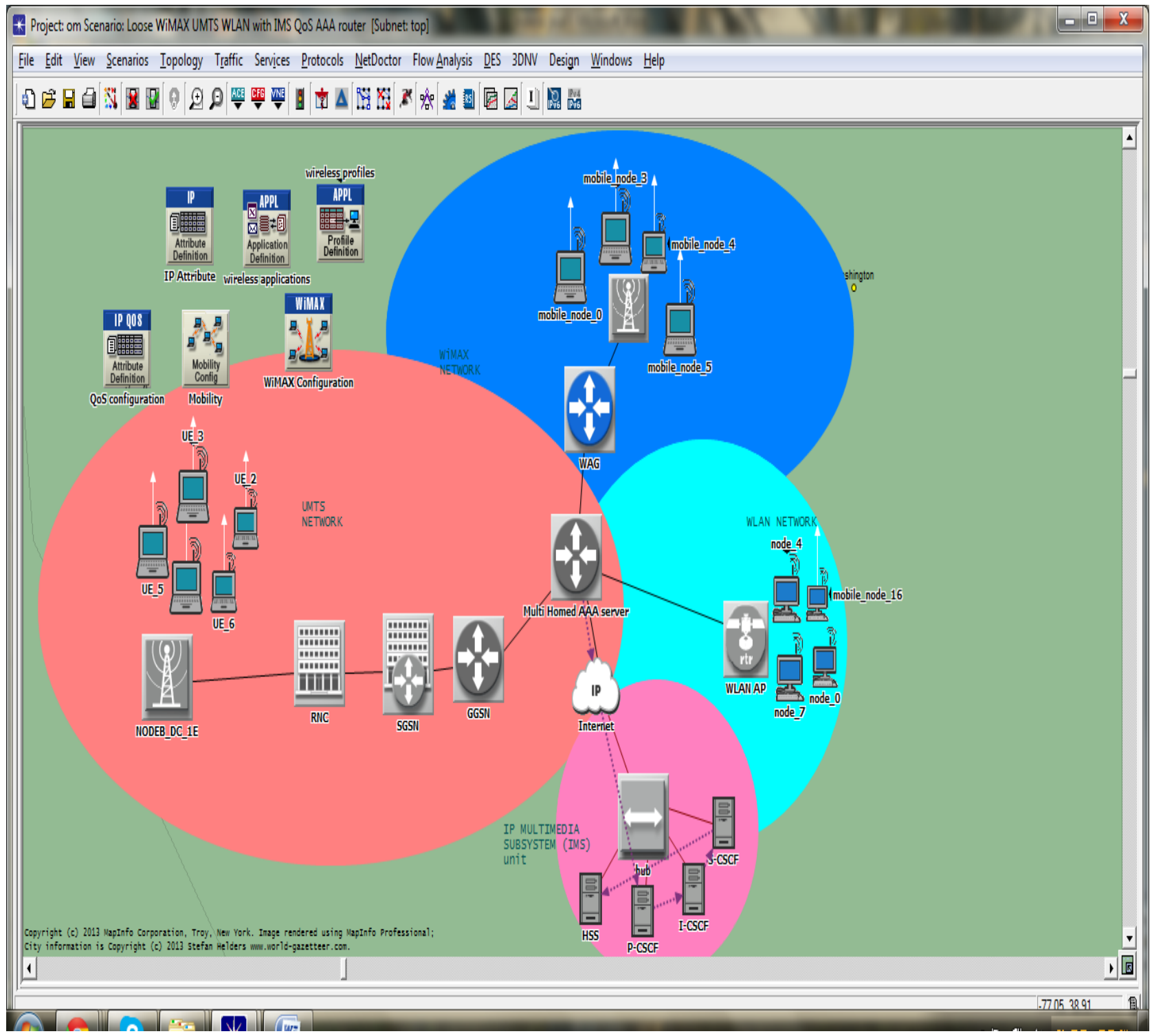

Figure 3 a: Loose coupled WiMAX-UMTS-WLAN interworking architecture with IMS

Here in the proposed loosely coupled WiMAX-UMTSWLAN, interworking with IMS, Base Station (BS) of WiMAX network is connected to Wireless access gateway (WAG) of WiMAX and in turn connected to Converging Serving Gateway (CSG) also called as AAA router. This Gateway can act as Multi-homed-CSG, i.e CSG-WiMAX for WiMAX network, CSG-WLAN for WLAN network and CSG-UMTS for UMTS network during interworking. CSGWiMAX performs several functions like that of local AAA server and handles mobility management. In the same way WLAN-Access point (AP) and UMTS-GGSN is also connected to same Gateway. It is assumed that MS is capable to support simultaneous communication with UMTS/WiMAX/WLAN and has prior knowledge about a WiMAX operator and WLAN operator which has interworking support and service agreement with its UMTS operator. Based on this information, MS scans for the operators' ID, registers with a selected BS and assigned a local IP address from the CSG-WiMAX/WLAN gateway. Then MS sends its identity information to CSGWiMAX/WLAN, which performs local authentication, Authorization and Accounting and sends MS's identity to the corresponding $3 \mathrm{G}$ operator's using the same CSG (AAA) [17]. If the authentication fails the MS will be disconnected, where 3GPP sends user profile to the common AAA server (CSG-WiMAX/WLAN), which performs mapping between MS's Local IP and Remote IP. At that moment, user will be able to access Internet through CSG-WiMAX/WLAN. In this work WiMAX-UMTS with IMS, WiMAX-UMTS-WLAN without IMS and WiMAX-UMTS-WLAN interworking architectures with IMS are compared for different QoS traffic delays. In Loose coupling WiMAX-UMTS-WLAN interworking architecture GGSN of UMTS network is indirectly connected to the WAG of WiMAX base station and WLAN access point router through internet backbone using common AAA server which will act as CSG for WiMAX/WLAN/UMTS. WiMAX is connected with the WLAN with the help of WLAN Access point. Common AAA serving gateway is connected to WiMAX-WAG, WLAN-AP and UMTS-GGSN and finally connected to internet backbone where IMS-SIP server's resides. IMS multimedia supporting server (SIP) session initiation protocol is connected to the Ethernet hub. Proxy-Call Session Control Function (P-CSCF), Serving-Call Session Control Function (S-CSCF) and 
Interrogating-Call Session Control Function (I-CSCF) is connected to the Hub. The proposed architecture connects UMTS Core Network where the SGSN, the GGSN and the AAA Server reside, a WLAN and a WiMAX network interconnected with the UMTS Core through specific functional entities and an IMS in charge of sessions' control. The Main goal of the proposed architecture is to place an IMS-AAA core on top of all technologies to manage unified and common control sessions. This new approach was driven by the fact that WiMAX offers a number of advantages compared to the UMTS' RAN (Radio Access Network) [1]. Firstly, it is a very cost efficient solution, in terms of deployment and maintenance. Secondly, it has better performance in terms of data rates in comparison with the UMTS RAN for bandwidth greedy applications (e.g. video calls). The biggest advantage that UMTS held against WiMAX was the mobility an coverage mechanism it offered. This disadvantage of the WiMAX has disappeared since in a latest release of the WiMAX mobility is supported. Also a WLAN access network was 'attached' to primary architecture following the architectural concept of [3] in order to enhance network's characteristics and technologies' coverage, and thus building a converged network with IMS technology users can access the $3 \mathrm{G}$ based services through the WiMAX and WLAN networks, since they are authenticated in the AAA Server and registered in the IMS core and served by SIP application servers. In the handoff procedures, the old PCSCF serving the user sends information concerning the active calls to the new P-CSCF thus reducing the latency needed for the handoff. The same P-CSCF serves the ANs' WAGs, whilst different S-CSCF and I-CSCF servers could be allocated for each AN [1]. The MSs are identified by multiple IP addresses and have the ability to choose the different Access Network that suits better the user requirements in terms of the type of the application to be activated, the connection costs, the AN's area coverage, the QoS, etc. For the successful registration to the IMS the public/private user identity, SIP support and DiffServ support is needed. A user or MS that wants to register to the IMS core through the UMTS network must first activate a PDP context. A PDP context is a record that is saved in the SGSN and the GGSN, and contains information about the user, as well as, about the active sessions. The record includes the user information and IP address, the quality of service parameters of the given session, etc. The PDP context activation procedure takes place after the GPRS (General Packet Radio Service) attach procedure. The procedure starts with the activate PDP context message sent by the user to the SGSN. The SGSN then queries the Domain Name Server (DNS) of the core network to find the GGSN to which the particular access point corresponds. The DNS replies with the GGSN's IP address and the SGSN forwards the PDP context activation request to the received IP [1]. The GGSN authenticates the subscriber and gets a dynamic IP address from the Dynamic Host Configuration Protocol (DHCP) server to assign it afterwards to the MS [1]. After that, the GGSN sends a create PDP context response to the SGSN. Finally the SGSN ends the procedure by sending an activate PDP context accept message to the user. At the end of this procedure, the user is ready to register to the IMS core with the SIP registration. As regards the WiMAX/WLAN network is concerned, each user that accesses the IMS or UMTS services must be registered in the HSS. In the same way when the MS of WiMAX wants to communicate with WLAN through IMS, here no need for PDP context activation Proxy-Call Session Control Function (P-CSCF), Serving-Call Session Control Function (S-CSCF) and Interrogating-Call Session Control Function (I-CSCF) is connected to the Hub. In order to configure IMS module, the $\mathrm{P}-\mathrm{CSCF}$ and S-CSCF proxy type is configured with diameter delay 0.005 Seconds and processing delay of 0.0001 Seconds. I-CSCF proxy server is configured with a diameter delay of 2.0 Seconds and processing delay of 0.5 Seconds. This would provide better QoS in turn for the overall architecture. Here Interactive, streaming and conversational and background applications are simulated for comparison. In addition hybrid QoS algorithm is also configured.

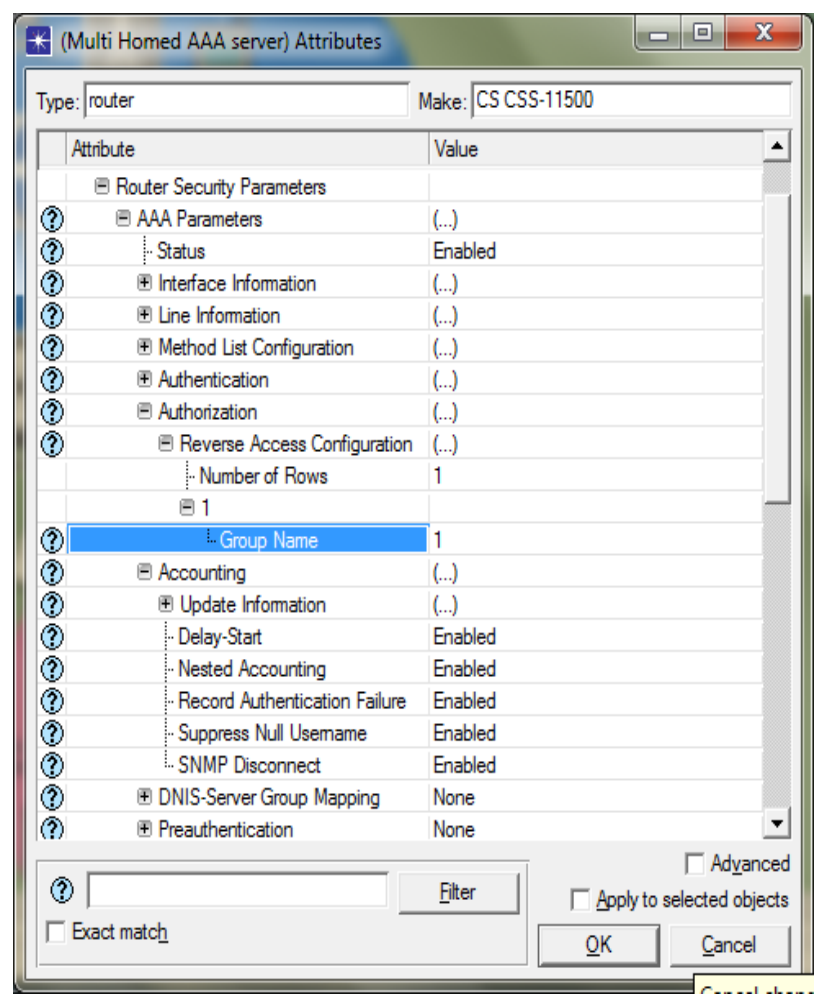

Figure 3 b: AAA serving gateways attributes modification

Figure $3 \mathrm{~b}$ shows AAA serving gateway attributes, where AAA parameters are configured. Here Authentication, Authorization and accounting parameters are configured and enabled for providing Security and better QoS.

\subsection{Loose coupled WiMAX-UMTS-WLAN integrating without IMS-SIP-AAA}




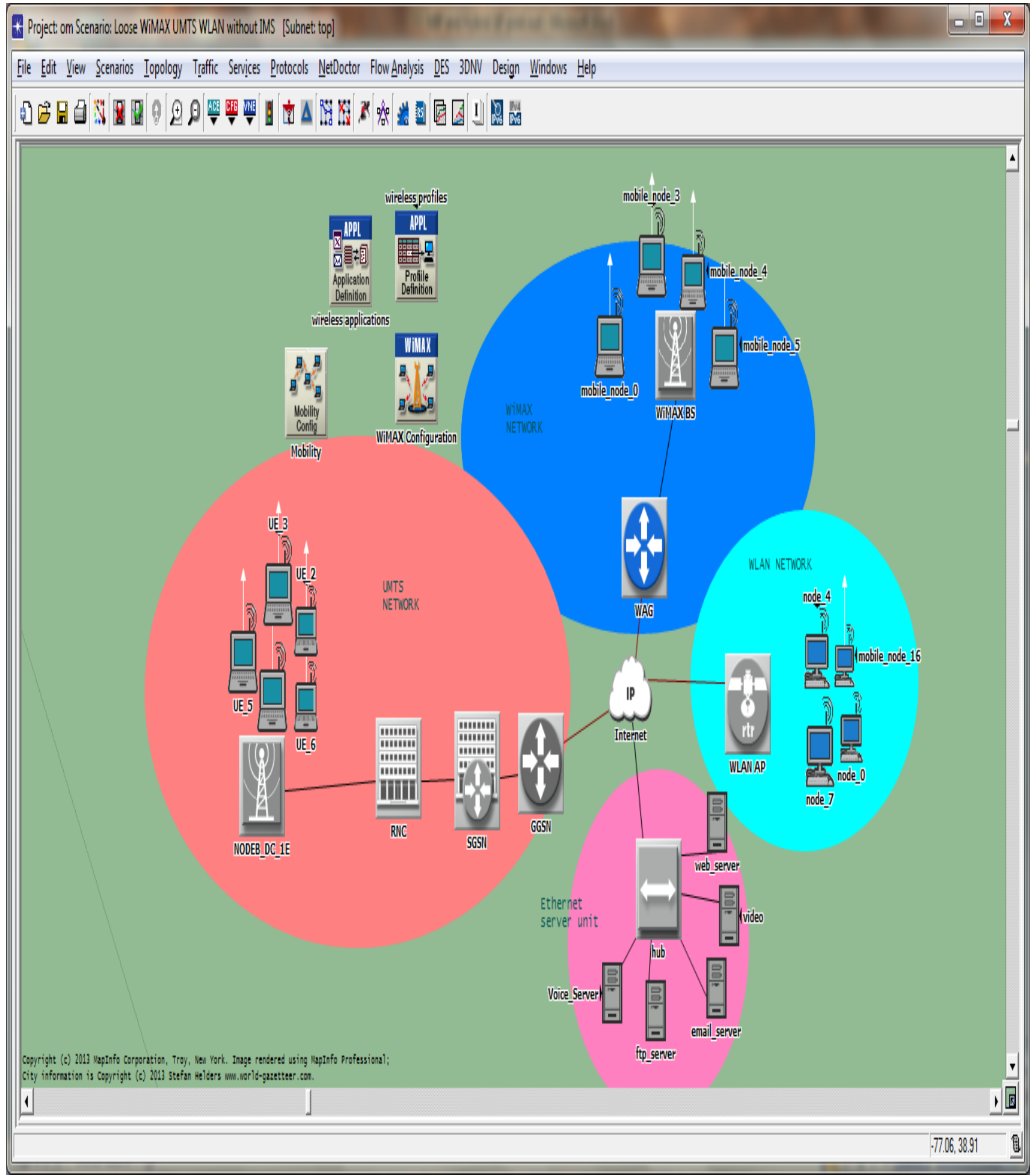

Figure 4: Loose coupled WiMAX-UMTS-WLAN integrated architecture without IMS-SIP-AAA and QoS

Figure 4 shows loosely coupled WiMAX-UMTS-WLAN integrated architecture without IMS and AAA serving gateway. Here IMS is not configured but ordinary Ethernet servers is connected at the hub for supporting various traffic such as VoIP, Video streaming, Web browsing and background traffic. The other connections are same like loosely coupled WiMAX-UMTS-WLAN with IMS. It is simulated to prove that WiMAX-UMTS-WLAN with IMS provide better QoS than without IMS.

\section{RESULTS AND DISCUSSIONS}

The proposed Loosely WiMAX-UMTS-WLAN interworking architecture with IMS is compared with Loosely WiMAXUMTS interworking architecture with IMS. In order to prove IMS signaling is used to improve QoS, loosely coupled WiMAX-UMTS-WLAN without IMS is also simulated and compared with above scenarios. Here Background traffic (email, FTP), Web browsing (Interactive multimedia), VoIP (Conversational), Video streaming (Streaming multimedia). 
The following results are shown below. The simulation time period taken for the analysis is 300 seconds ( $\mathrm{X}$ axis) common for all the graphs. $\mathrm{Y}$ axis shows the corresponding QoS simulation parameters. Figure 5a shows Email upload response time in sec for loose coupled WiMAX-UMTS with IMS and Loose coupled WiMAX-UMTS-WLAN with IMS and without IMS. It is observed that upload response time taken by Loose Coupled WiMAX-UMTS-WLAN Architecture with IMS is very low when compared to Loose coupled WiMAX-UMTS Architecture with IMS. This clearly shows delay is very low in the interworking WiMAX-UMTSWLAN Architecture with IMS.
Figure $5 \mathrm{~b}$ shows the comparison of email download response time in sec taken by Loose Coupled WiMAX-UMTS-WLAN Interworking Architectures with IMS and without IMS and Loose coupled WiMAX-UMTS with IMS. It is observed that Email download response time taken by Loose coupled WiMAX-UMTS-WLAN Interworking Architecture with IMS is very low when compared to Loose coupled WiMAXUMTS interworking architecture with IMS and WiMAXUMTS-WLAN without IMS.

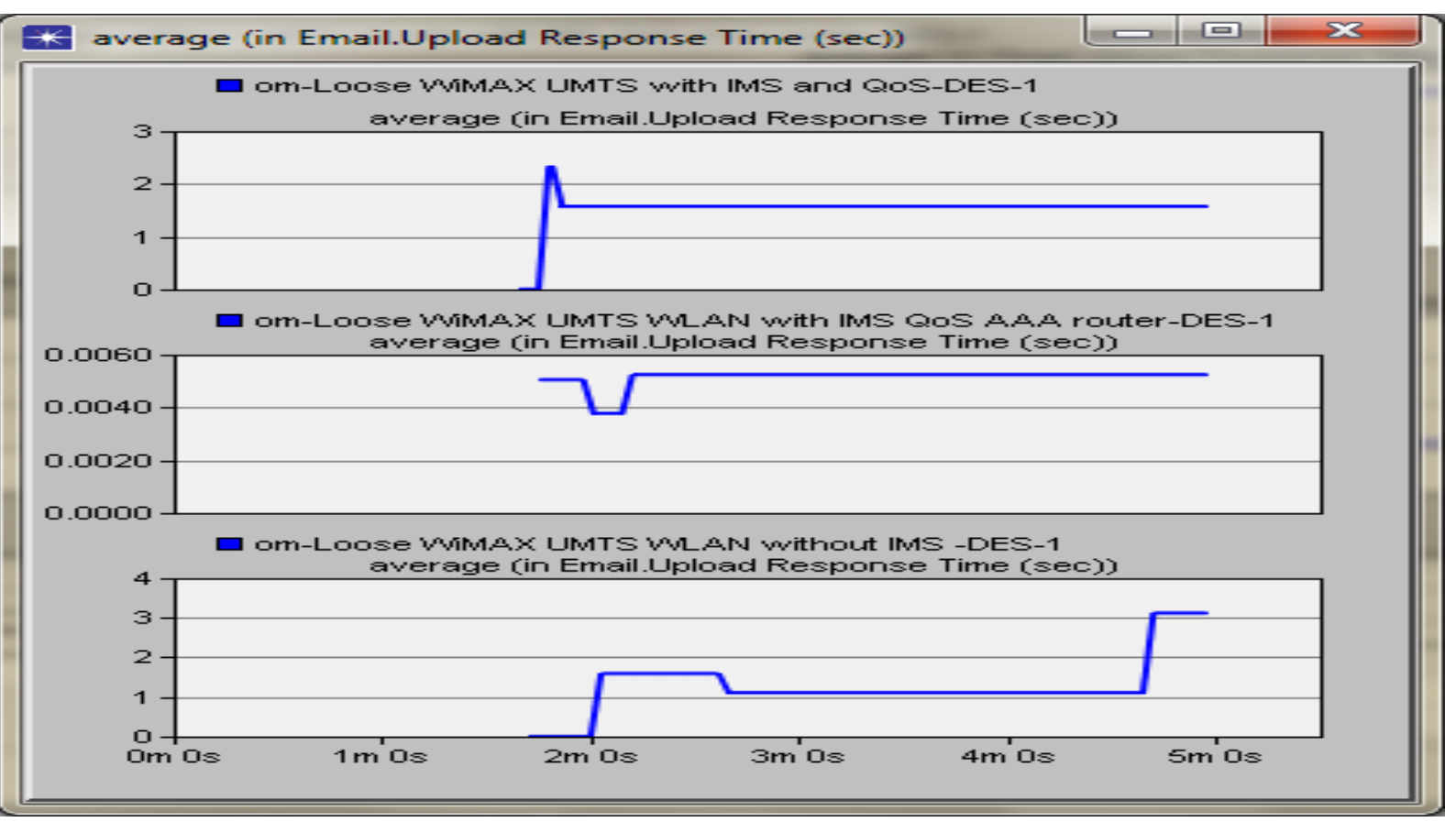

Figure 5a: Email upload response time (sec) for Loose coupled WiMAX-UMTS with IMS and Loose coupled WiMAX-UMTSWLAN with IMS and without IMS

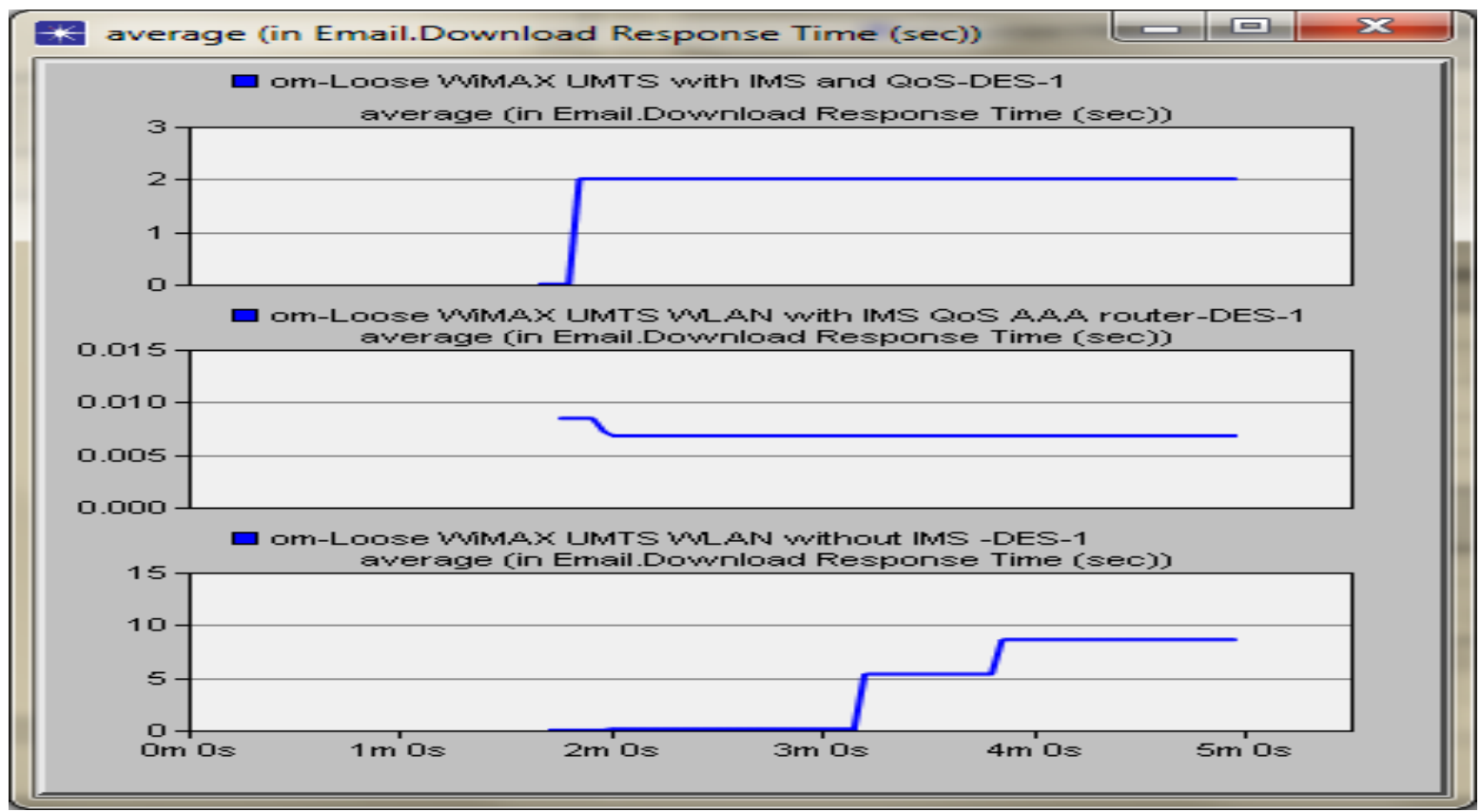

Figure 5b: Email download response time (sec) for Loose coupled WiMAX-UMTS with IMS and Loose coupled WiMAXUMTS-WLAN with IMS and without IMS 


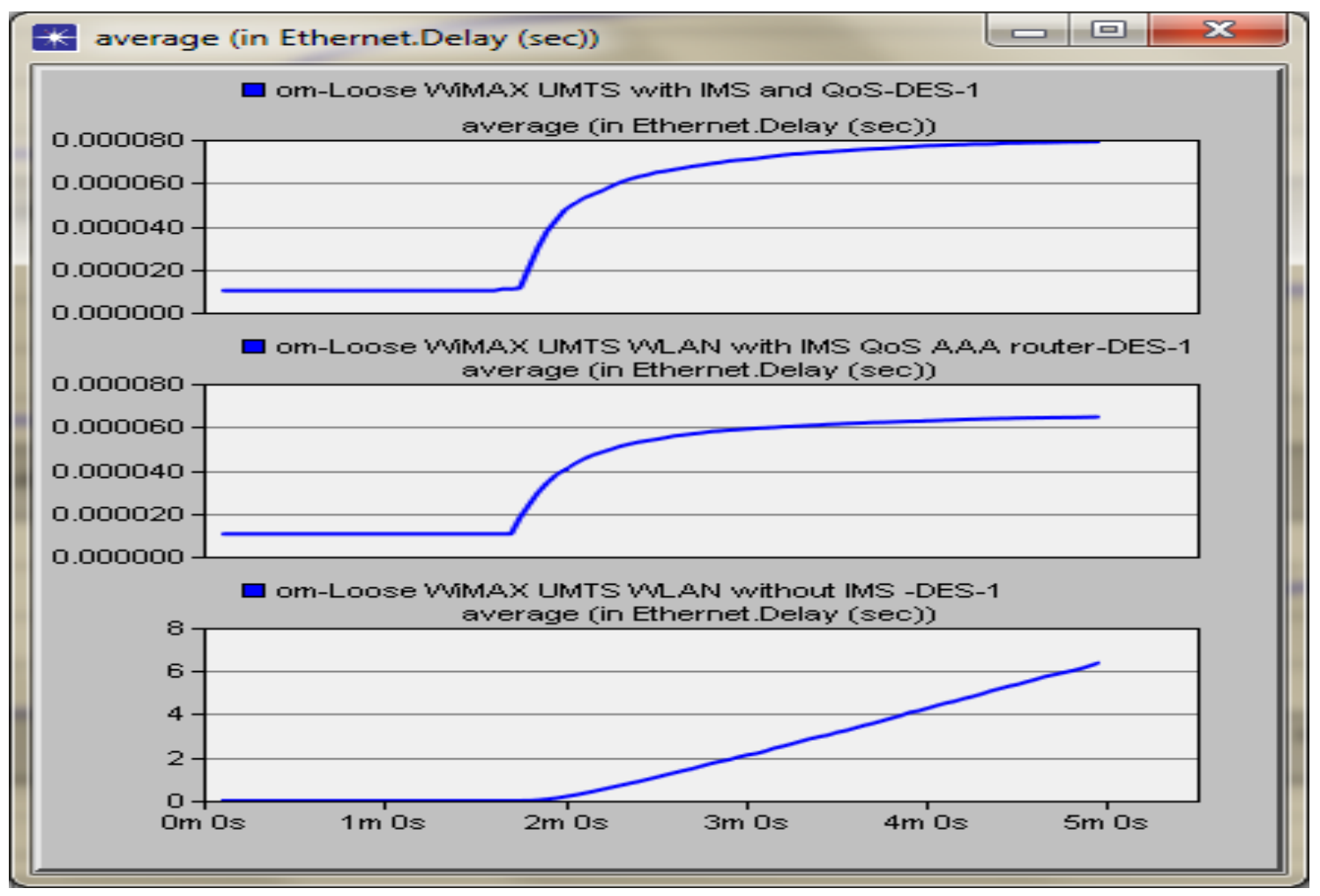

Figure 6: Ethernet Delay (sec) for Loose coupled WiMAX-UMTS with IMS and Loose coupled WiMAX-UMTS-WLAN with IMS and without IMS

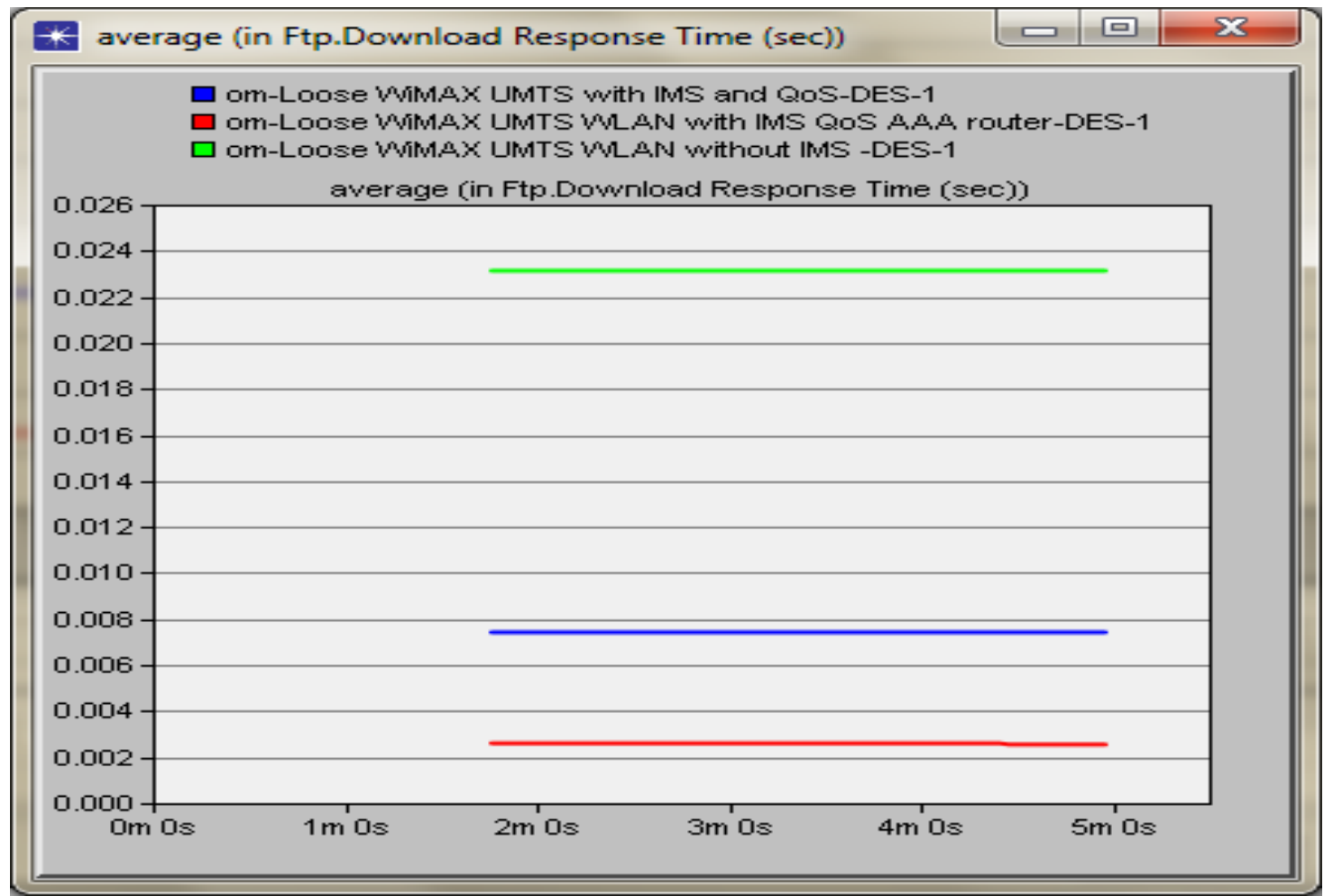

Figure 7a: FTP download response time (sec) for Loose coupled WiMAX-UMTS with IMS and Loose coupled WiMAXUMTS-WLAN with IMS and without IMS 


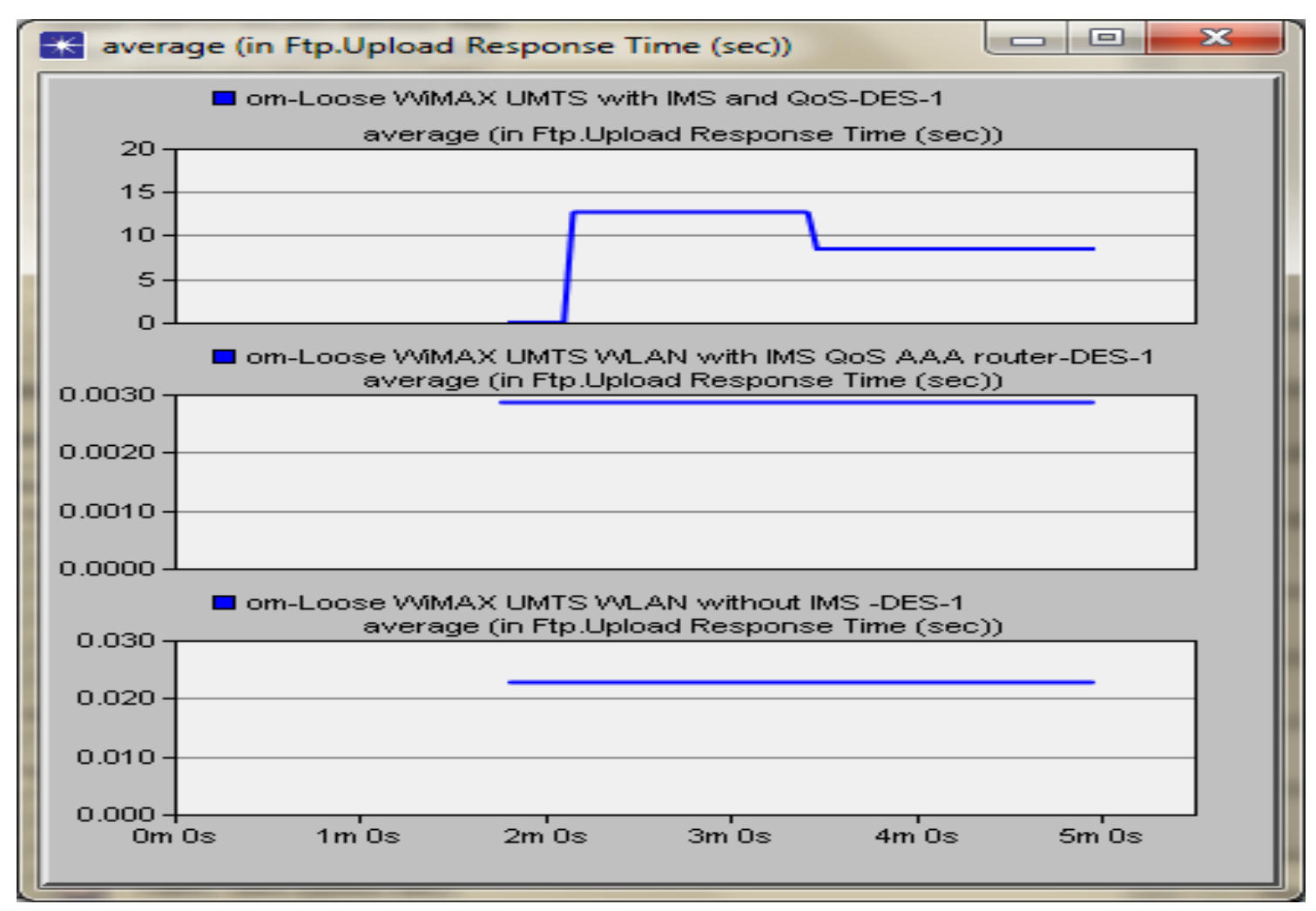

Figure 7b: FTP upload response time (sec) for Loose coupled WiMAX-UMTS with IMS and Loose coupled WiMAX-UMTSWLAN with IMS and without IMS

Figure 6 shows Ethernet delay for Proposed WiMAX-UMTSWLAN with IMS is very low nearing 0.000060 seconds at the end of the simulation whereas the same delay for WiMAXUMTS with IMS is 0.000080 seconds. WiMAX-UMTSWLAN with IMS is having larger Ethernet delay of 6 seconds. Figure $7 \mathrm{a}$ and $7 \mathrm{~b}$ shows FTP download response and
Upload response for WiMAX-UMTS-WLAN with IMS and without IMS and WiMAX-UMTS with IMS. It is clearly shown from the figure; both download response and upload response is very low for proposed WIMAX-UMTS-WLAN with IMS.

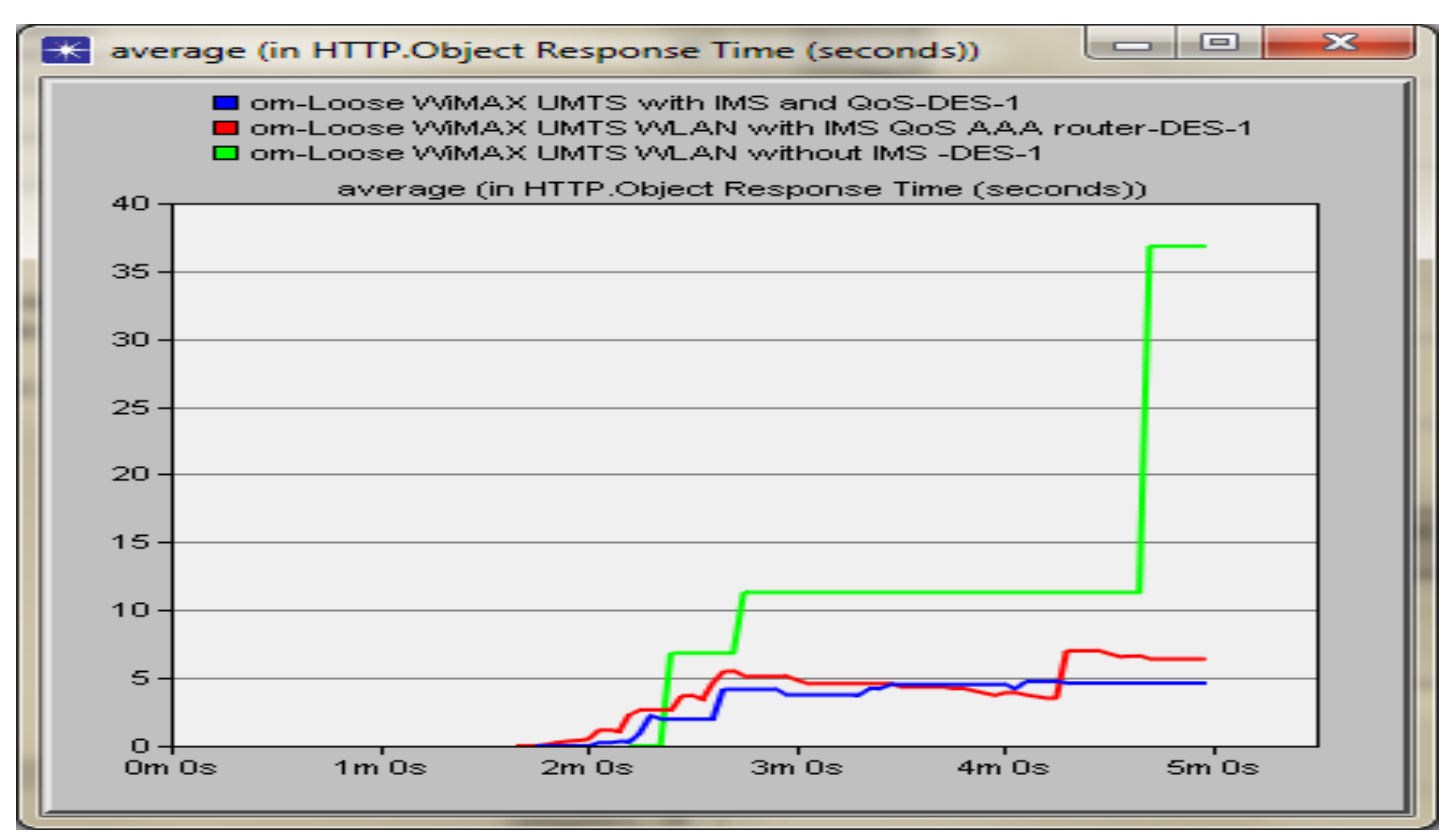

Figure 8a: HTTP Object response time (sec) for Loose coupled WiMAX-UMTS with IMS and Loose coupled WiMAX-UMTSWLAN with IMS and without IMS 


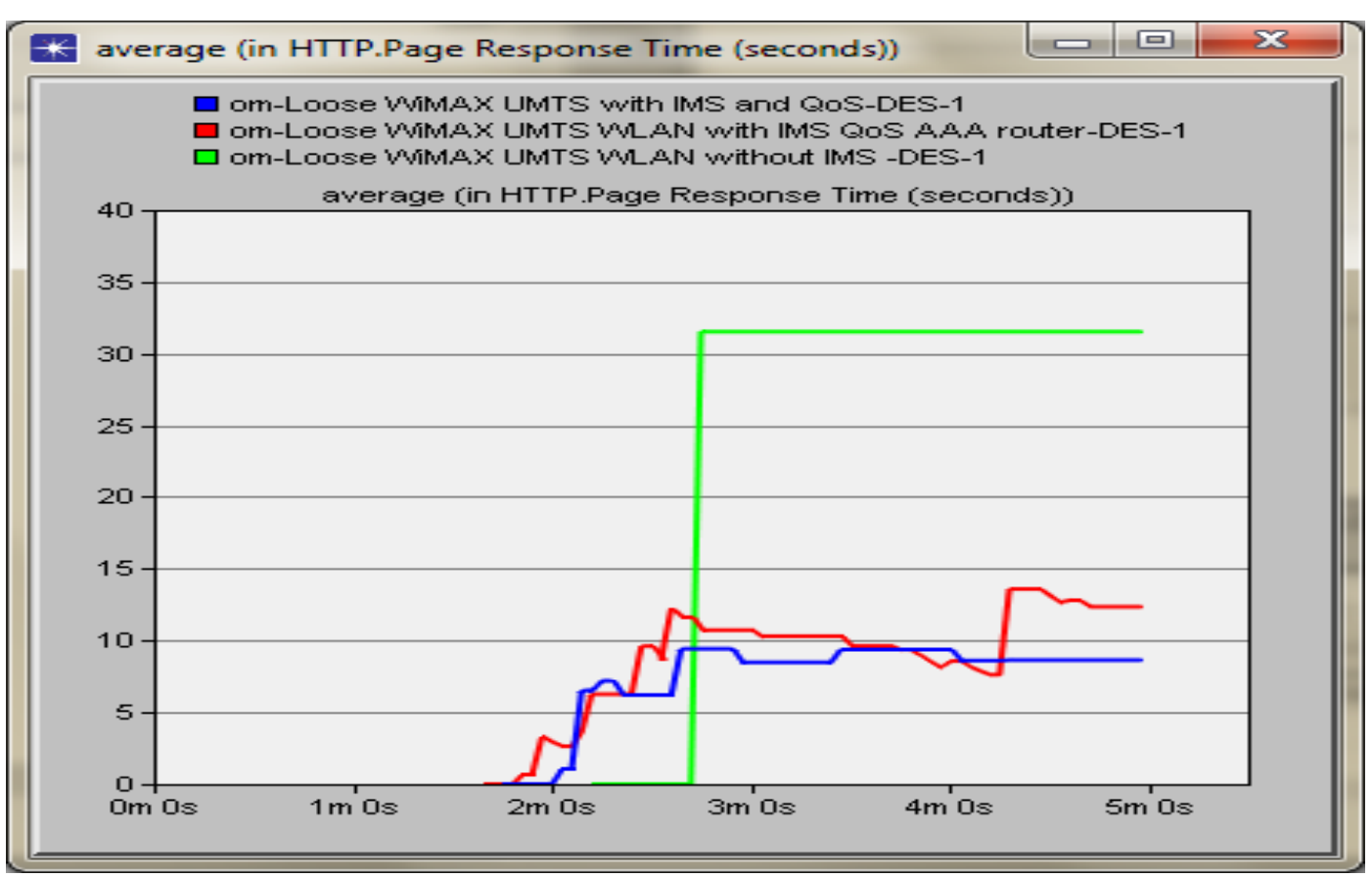

Figure 8b: HTTP Page response time (sec) for Loose coupled WiMAX-UMTS with IMS and Loose coupled WiMAX-UMTSWLAN with IMS and without IMS

Figure $8 \mathrm{a}$ and $8 \mathrm{~b}$ shows HTTP (Interactive multimedia) Object response time (sec) and page response time (sec) for loose coupled WiMAX-UMTS with IMS and Loose coupled WiMAX-UMTS-WLAN with IMS and without IMS. Here Page response and Object response are more or less same for WiMAX-UMTS with IMS and WiMAX-UMTS-WLAN with
IMS, but the traffic received for loosely coupled WiMAXUMTS-WLAN is very high when compared to loosely coupled WiMAX-UMTS for the same HTTP response delay. It is clearly shown in figure $8 \mathrm{c}$. Since three networks are interworked, there will be little more delay than two network architecture

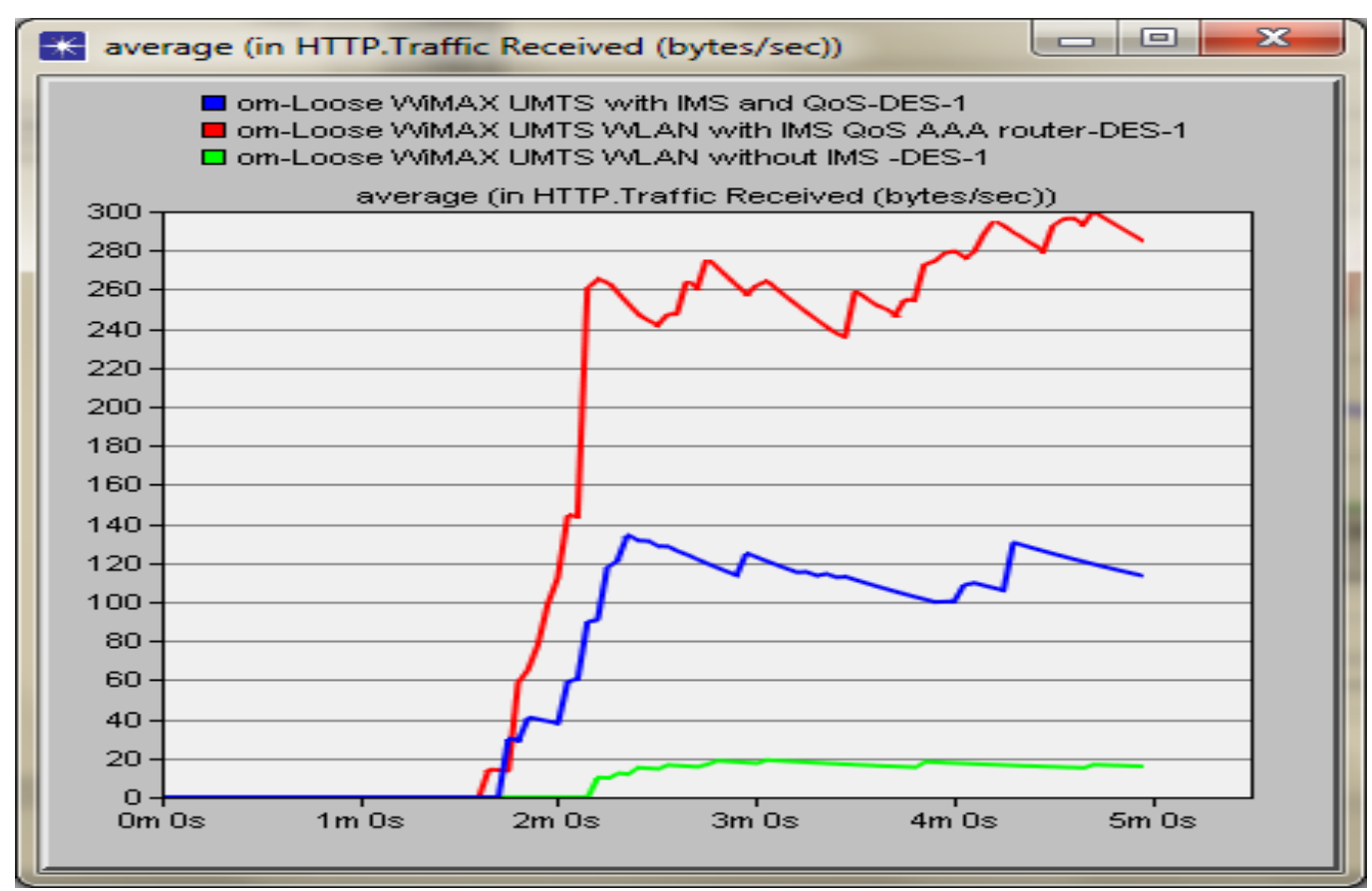

Figure 8c: HTTP Traffic received (Bytes/sec) for Loose coupled WiMAX-UMTS with IMS and Loose coupled WiMAX-UMTSWLAN with IMS and without IMS 


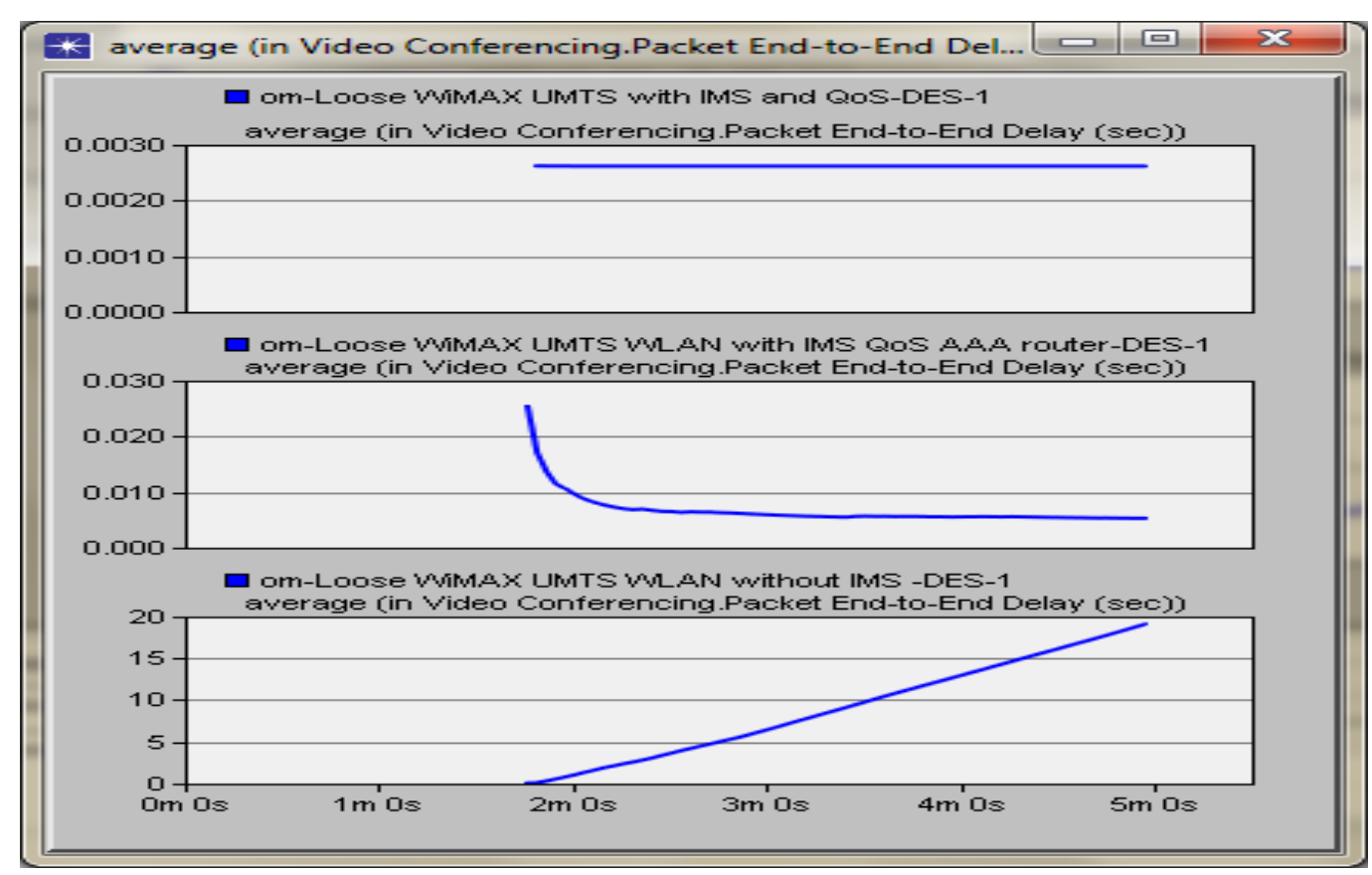

Figure 9a: Video Streaming Packet End-to-End Delay (sec) for loose coupled WiMAX-UMTS with IMS and Loose coupled WiMAX-UMTS-WLAN with IMS and without IMS

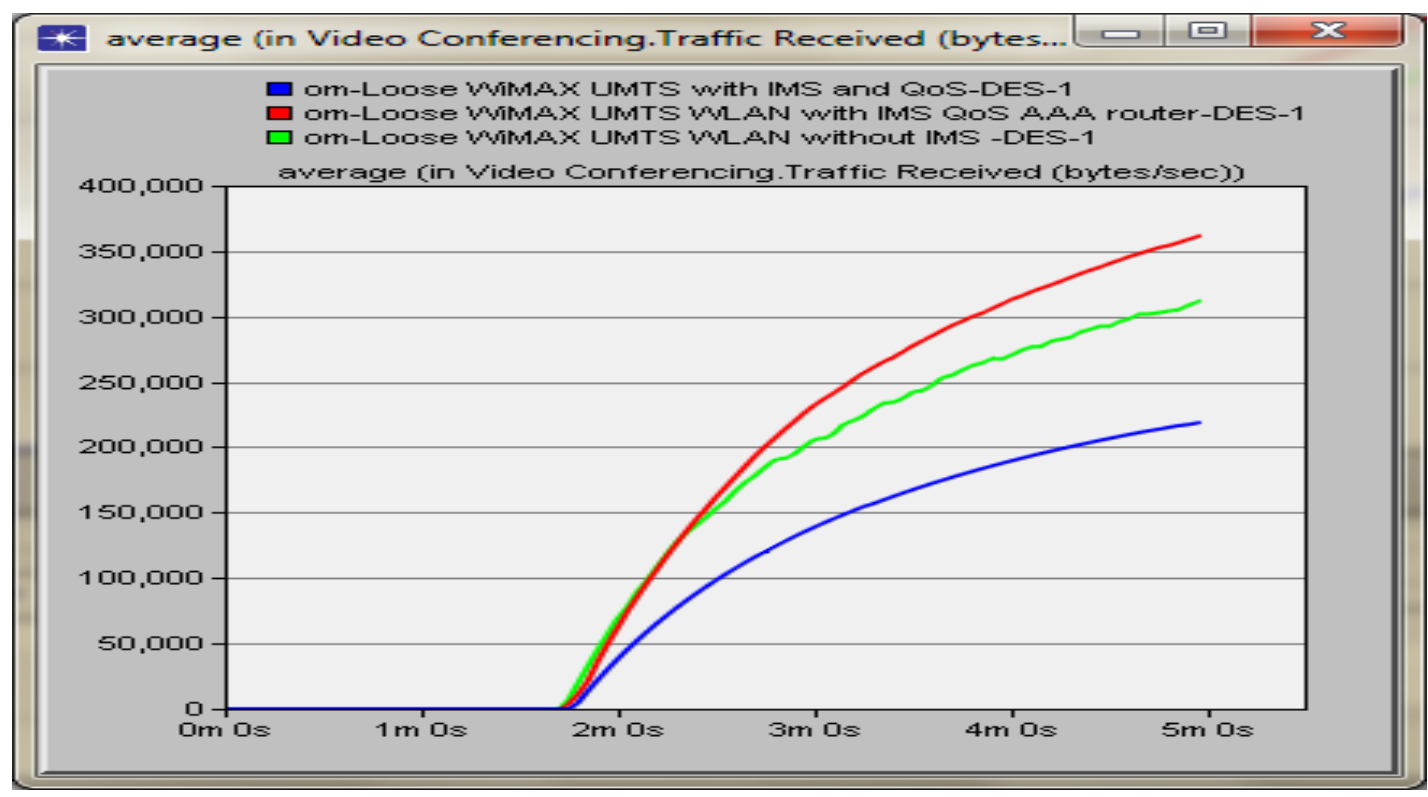

Figure 9b: Video traffic received (Bytes/sec) for Loose coupled WiMAX-UMTS with IMS and Loose coupled WiMAX-UMTSWLAN with IMS and without IMS

Figure 9a shows Video streaming End-to-End Delay during vertical handover for loose couple WiMAX-UMTS with IMS interworking. The delay is very low for WiMAX-UMTS with IMS $(2 \mathrm{~ms})$ at the end of simulation. The video streaming traffic received for the same delay for WiMAX-UMTS with IMS is very low. It is shown in figure $9 \mathrm{~b}$. The traffic received for WiMAX-UMTS-WLAN with IMS is large than without IMS and WiMAX-UMTS with IMS. The delay is also very less nearly $5 \mathrm{~ms}$ at the end of simulation time of $300(\mathrm{sec})$ with more video traffic. This shows proposed loosely coupled WiMAX-UMTS-WLAN with IMS outperforming well in terms of traffic with affordable delay. 


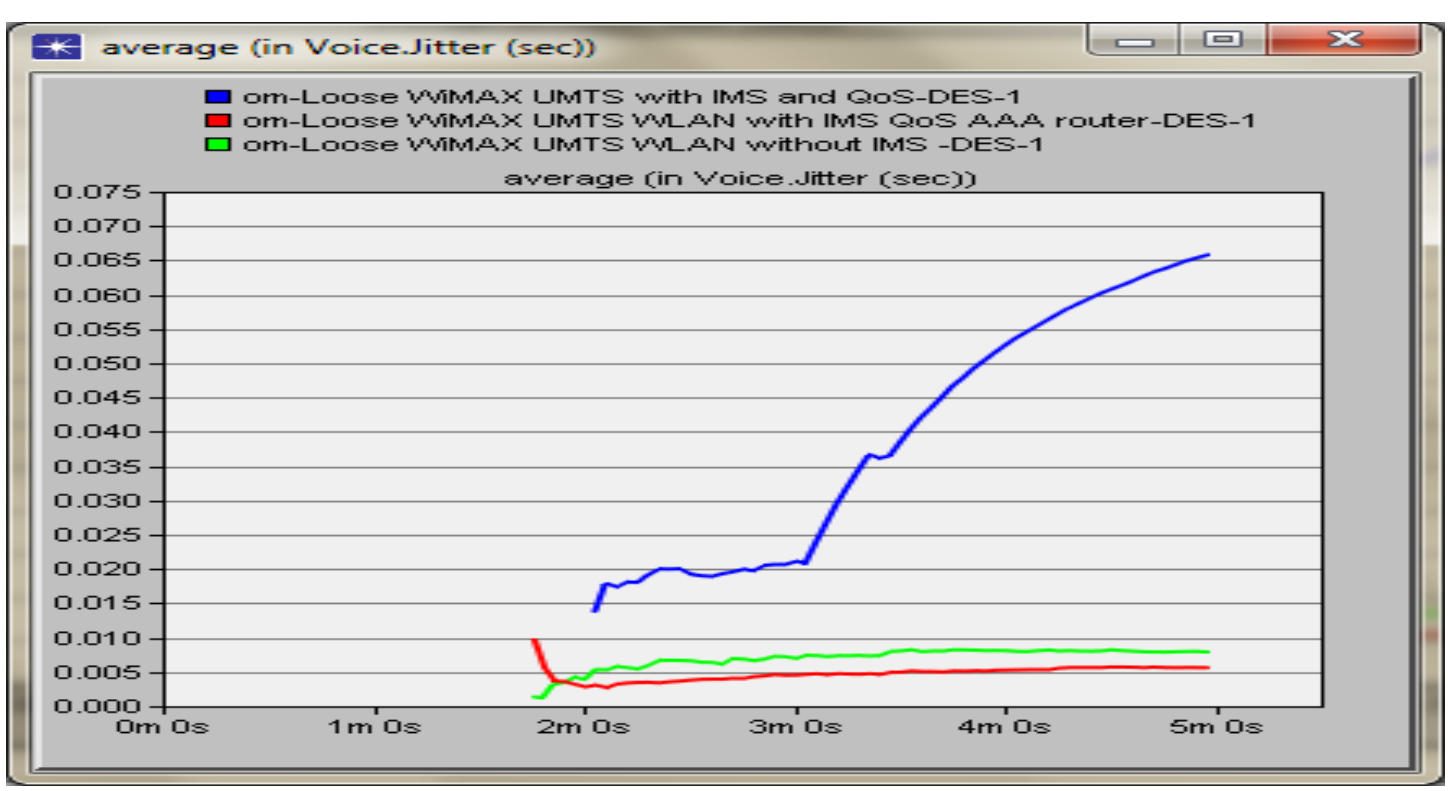

Figure 10 a: Voice Jitter (sec) for Loose coupled WiMAX-UMTS with IMS and Loose coupled WiMAX-UMTS-WLAN with IMS and without IMS

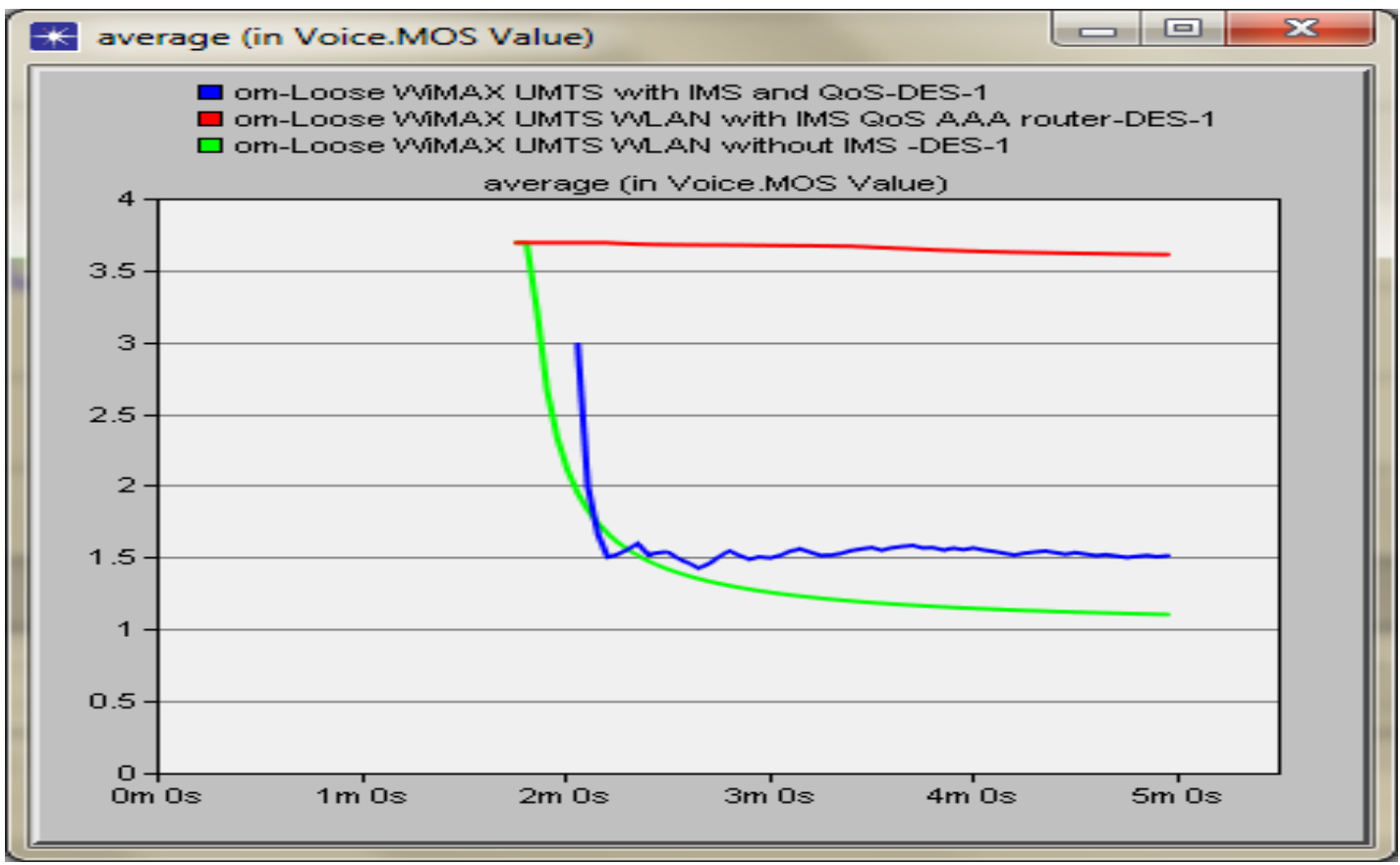

Figure 10 b : Voice MOS (Mean Opinion Score) for Loose coupled WiMAX-UMTS with IMS and Loose coupled WiMAXUMTS-WLAN with IMS and without IMS

Figure 10a shows VoIP Jitter in seconds compared for all three architectures. Voice Jitter for Loosely coupled WiMAXUMTS-WLAN with IMS and without IMS is low when compared to WiMAX-UMTS interworking with IMS. Jitter value is for WiMAX-UMTS-WLAN with IMS is 2 ms and 5 ms or WiMAX-UMTS-WLAN without IMS. The Delay for WiMAX-UMTS with IMS is $65 \mathrm{~ms}$ at the end. The Figure 10 b shows Voice Mean opinion Score (MOS). In Mobile communications mean opinion score (MOS) provides a numerical measure of the voice quality. The MOS value range between 1 to 5 , where lowest perceived audio quality is given by the range 1 , and the highest perceived audio quality measurement lies in the range 5 but in practical MOS value of 4 is the typical value for mobile communication. It is higher for WiMAX-UMTS-WLAN with IMS (3.9) than other two architectures. This clearly shows Proposed architecture outperforms well both in terms of delay and traffic. 


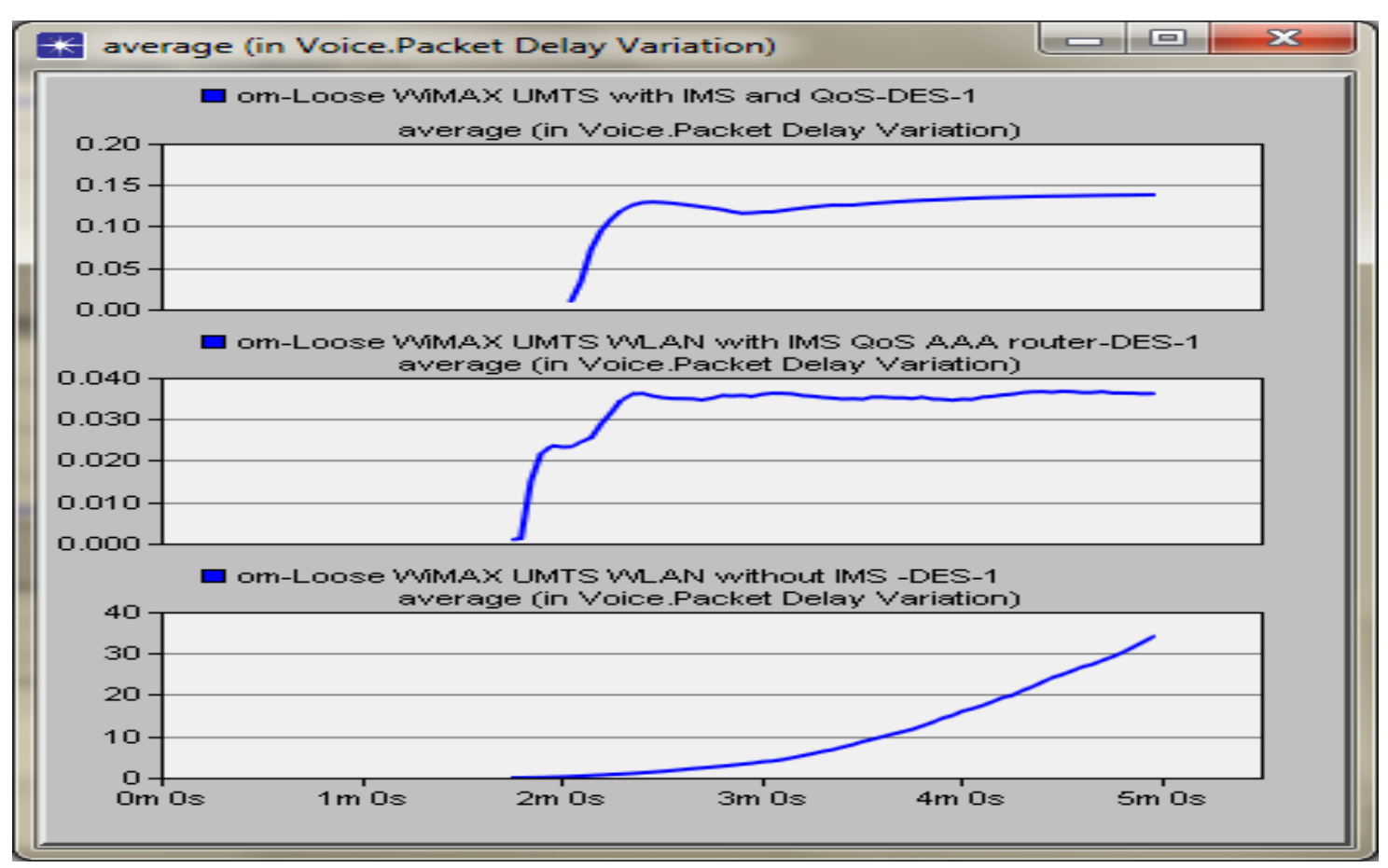

Figure 10 c: Voice Packet Delay Variation (sec) for Loose coupled WiMAX-UMTS with IMS and Loose coupled WiMAXUMTS-WLAN with IMS and without IMS

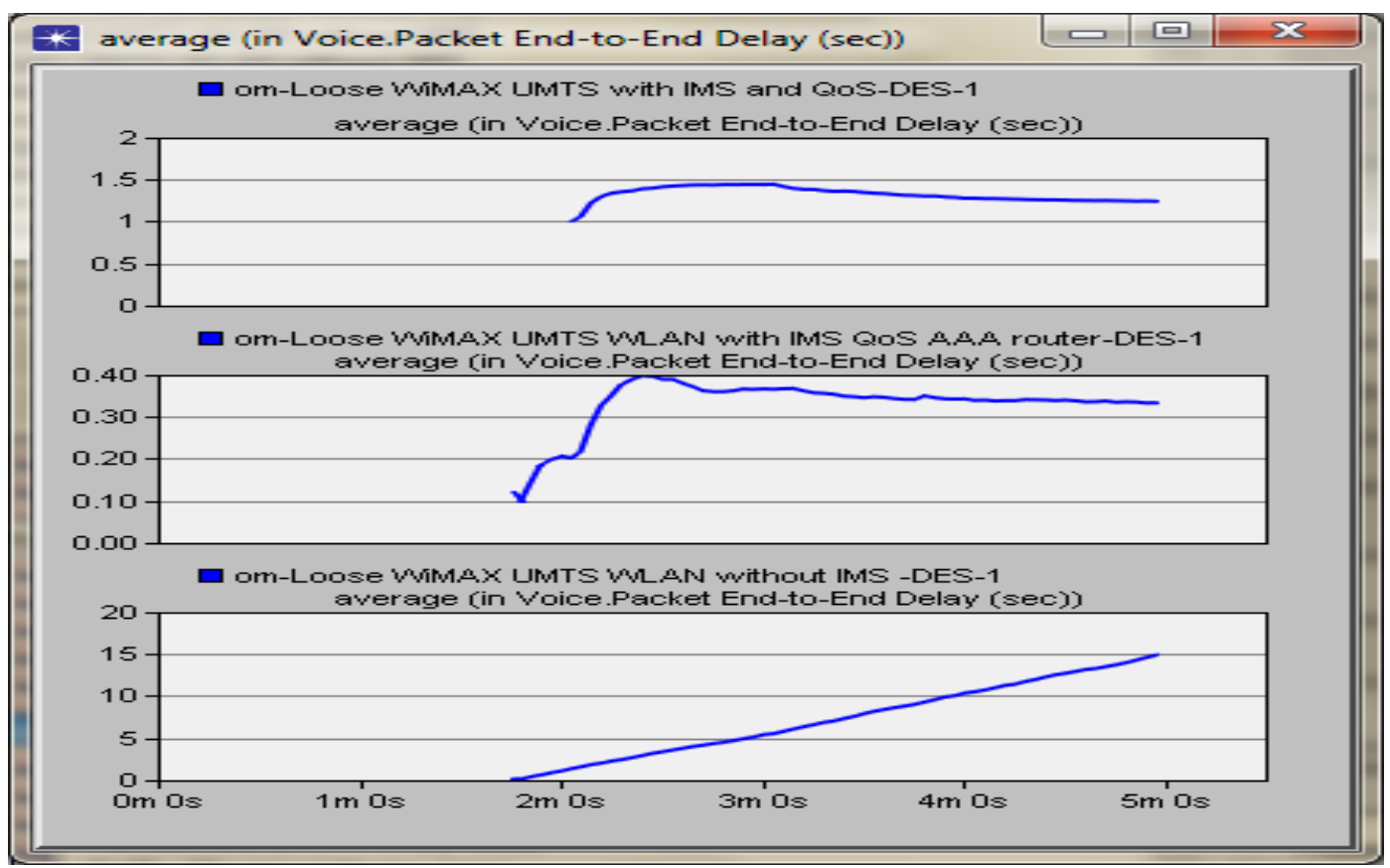

Figure 10 d: Voice Packet End-to-End Delay (sec) for Loose coupled WiMAX-UMTS with IMS and Loose coupled WiMAXUMTS-WLAN with IMS and without IMS

In figures 10c Voice packet delay variations of WiMAXUMTS-WLAN with IMS and Without IMS and WiMAXUMTS with IMS is shown. It is clear from the figure loosely coupled WiMAX-UMTS-WLAN with IMS having less Delay. In figure $10 \mathrm{~d}$ Voice Packet End-to-End delays is shown. It is also very less for the proposed WiMAX-UMTSWLAN with IMS. The end-to-end delay metric investigated for the WiMAX, UMTS and WLAN interfaces is dependent on the end-to-end VoIP signaling and data paths, the codec, and the payload size of the packets. The voice Packet End-to
End delay for WiMAX-UMTS with IMS is 1.5 seconds, and for WiMAX-UMTS-WLAN with IMS are $300 \mathrm{~ms}$. It is 15 seconds for WiMAX-UMTS-WLAN without IMS. The traffic received is shown in figure 10e. The Voice traffic received for WiMAX-UMTS-WLAN without IMS is slightly higher than with IMS but with more delay nearing 30 seconds of packet delay variation at the end of simulation. The same delay for WiMAX-UMTS-WLAN with IMS is $35 \mathrm{~ms}$ and for WiMAXUMTS with IMS are $150 \mathrm{~ms}$. The traffic received is shown in figure $10 \mathrm{e}$. 


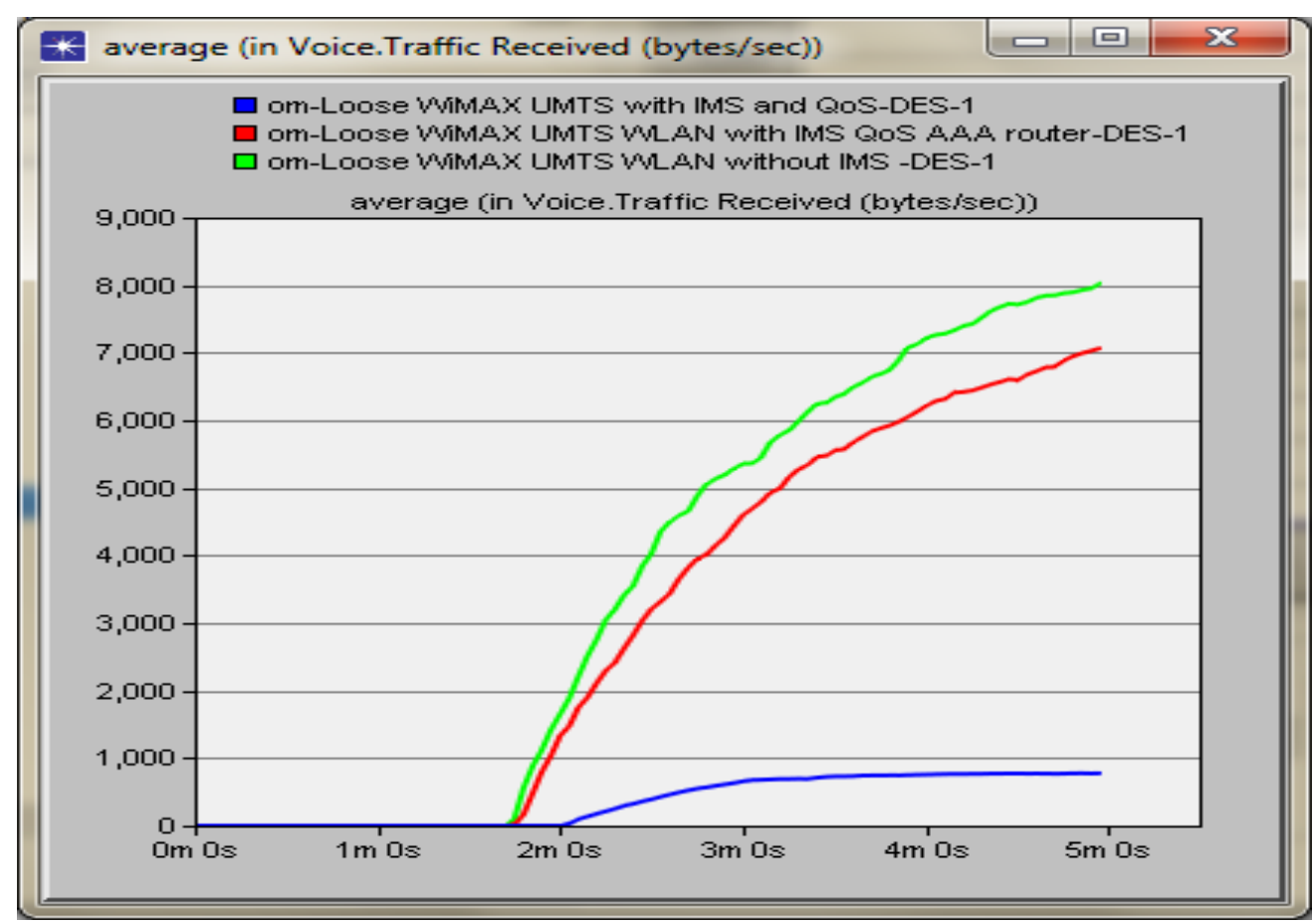

Figure 10 e: Voice traffic received (Bytes/sec) for Loose coupled WiMAX-UMTS with IMS and Loose coupled WiMAXUMTS-WLAN with IMS and without IMS

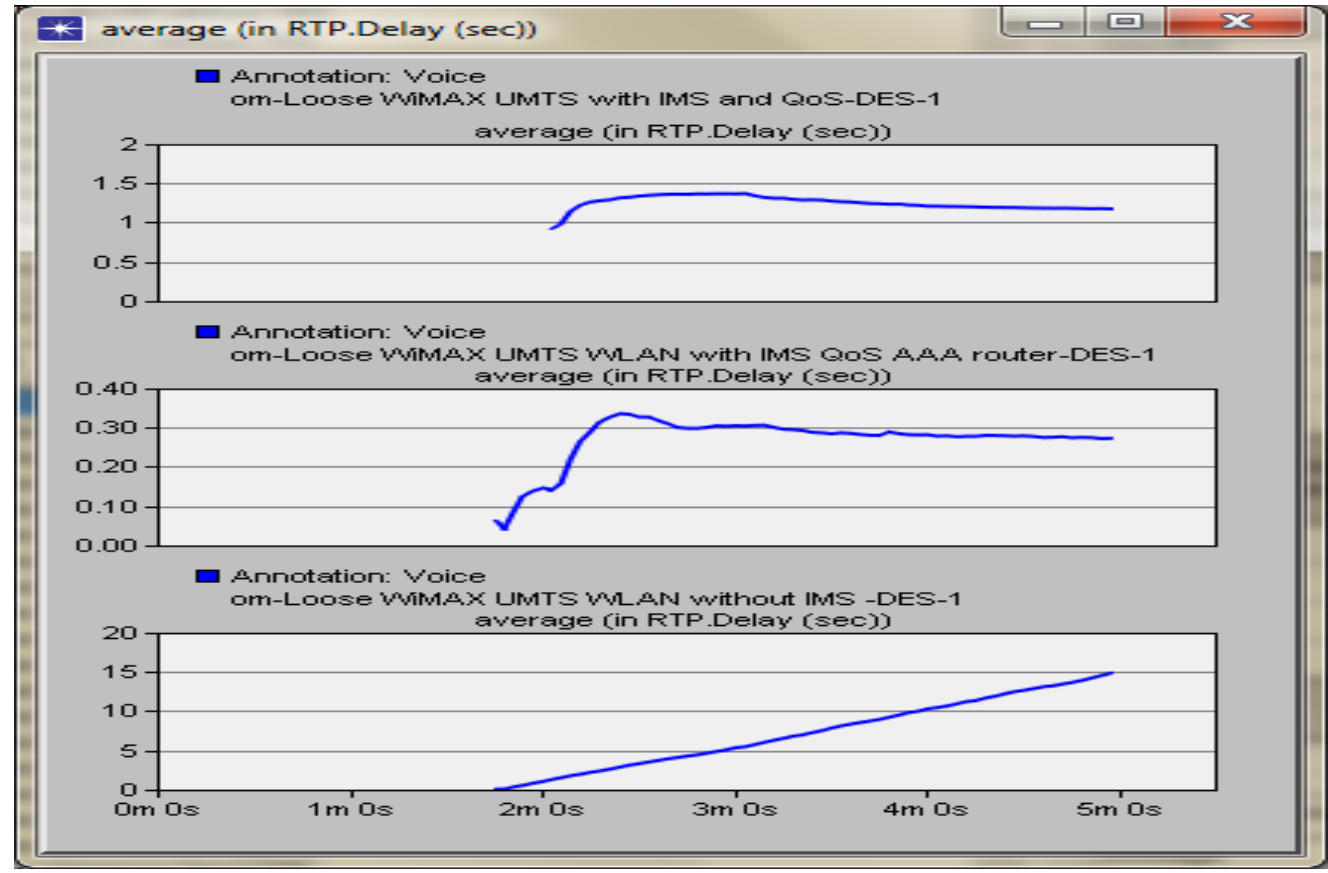

Figure 11 a: Real time Protocol Delay for Loose coupled WiMAX-UMTS with IMS and Loose coupled WiMAX-UMTS-WLAN with IMS and without IMS

The IMS uses the Real Time Protocol (RTP) for transporting real time audio and video over the User Datagram Protocol (UDP) as the transport protocol. It further uses the Real Time Control Protocol (RTCP) for providing the RTP with QoS statistics and information to provide intermediary synchronization on the media stream. The Session Initiation Protocol is used to initiate a session between two endpoints. SIP does not carry any voice or video data itself - it merely allows two endpoints to set up connection to transfer that traffic between each other via the Real-time Transport
Protocol (RTP). SIP Protocol used for establishing a session (call). IP address and port information is exchanged. RTP - After SIP establishes a session, this protocol is used for exchanging voice packets. The SIP protocol is a description and signaling protocol used to describe, establish, modify and terminate multimedia sessions or calls. SIP uses an IP network to transport the signaling messages through UDP and TCP. In the proposed model SIP session establishment is shown using UDP. Hence RTP delay and UDP session delay is simulated for SIP session Management. 


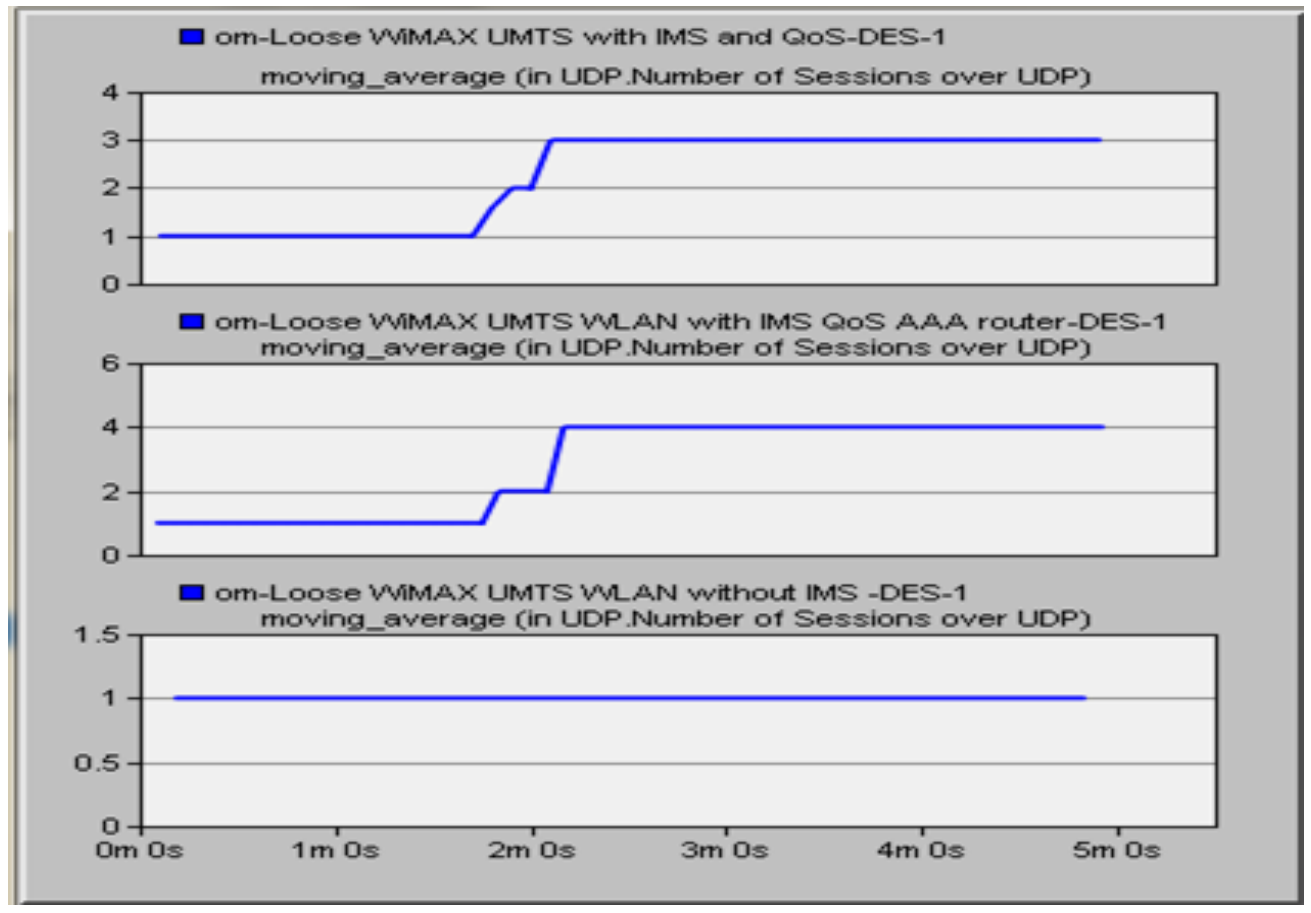

Figure 11 b: Number of sessions in SIP using UDP for Loose coupled WiMAX-UMTS with IMS and Loose coupled WiMAXUMTS-WLAN with IMS and without IMS

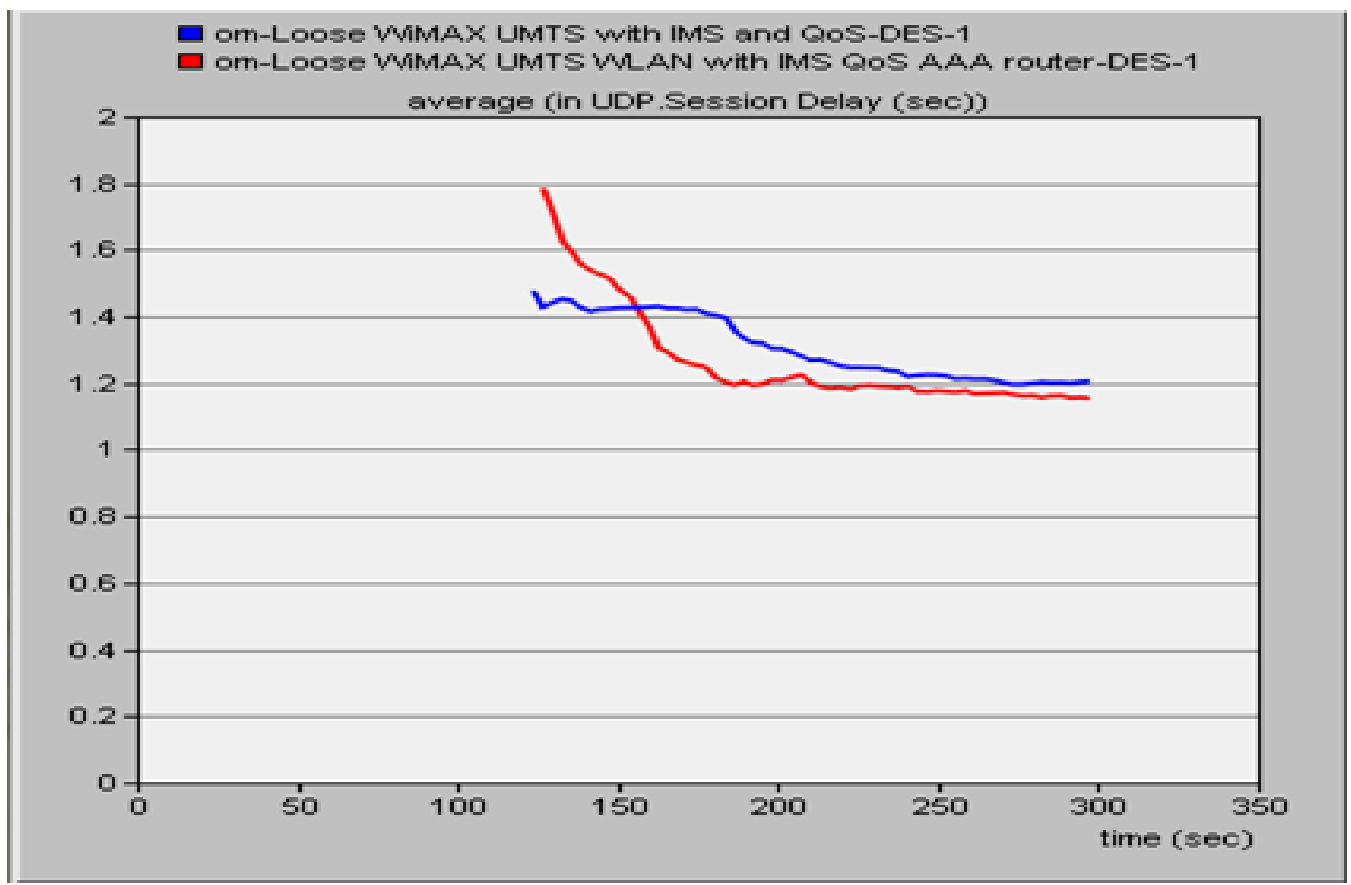

Figure 11c : Session Delay in SIP using UDP for Loose coupled WiMAX-UMTS with IMS and Loose coupled WiMAXUMTS-WLAN with IMS and without IMS

Figure 11a shows RTP delay using SIP for VoIP. Here RTP delay for WiMAX-UMTS-WLAN with IMS is very small compared to other two architectures. Here for without IMS the delay is very larger reaching 15 seconds. Hence it is clearly shown that IMS improves QoS performance in terms of delay in real time. Figure $11 \mathrm{~b}$ shows Number of sessions during handover. Number of sessions support is more for WiMAXUMTS-WLAN with IMS. The session delay is shown in figure 11c. The session delay is shown for SIP-IMS architectures. Hence WiMAX-UMTS-WLAN without IMS is not having SIP session establishment. The Session delay for WiMAX-UMTS-WLAN with IMS is comparatively low.

\section{CONCLUSION}

In this paper a loose coupled interworking model that integrates a WiMAX network, a UMTS network and a WLAN is proposed in an IMS compatible architecture. The main idea for the proposed architecture was to converge UMTS 
network, WiMAX network and WLAN network and to place an IMS-AAA serving core on top of both technologies to manage sessions to improve QoS. This approach was driven by the fact that the WiMAX if it is interworked with the already established 3G cellular networks (e.g. UMTS) and WLAN it is foreseen that it will form an important part of a future $4 \mathrm{G}$ network. IMS networks are still in an ongoing activity with the industry and the research community to constantly trying to solve open issues concerning session control, authorization, authentication, Quality of Service (QoS), charging, personal mobility, etc.. The proposed architecture with proposed algorithm outperforms well for different types of traffics.

\section{REFERENCES}

[1] Psimogiannos, N., Sgora, A., and Vergados, D. D. 2011. An IMS-based network architecture for WiMAX-UMTS and WiMAX-WLAN interworking, Computer Communications, (2011), 34, (9), pp. 1077-1099.

[2] 3GPP TS 23.228, IP multimedia subsystem (IMS), Version 8.7.0, Release 8,(2008).

[3] Rosenberg, J.et al., SIP: Session Initiation Protocol, IETF RFC 3261, and (June 2002).

[4] Agrawal, P., Hung, J.,Yeh, Chen, J.C., and Zhang, T. (2008). IP multimedia subsystems in 3GPP and 3GPP2: overview and scalability issues, IEEE Communications Magazine, 138-145.

[5] Postel, J. (1981). Internet Protocol, IETF RFC 791, September.

[6] Camarillo, G., and Garcia-Martin, M.A. (2006). The 3G IP Multimedia Subsystem (IMS),Secondsond ed., Wiley.

[7] Calhoun, P. et al., (2003). Diameter Base Protocol, IETF RFC 3588, September.
[8] Rigney, C., Willens, S., Rubens, A., and Simpson, W. (2000). Remote Authentication Dial In User Service (RADIUS), IETF RFC 2865, June.

[9] Kent, S., Atkinson, R. (1998). Security Architecture for the Internet Protocol, IETF RFC2401, November.

[10] Dierks, T., and Allen, C. (1999). The TLS Protocol Version 1.0, IETF RFC 2246, January.

[11] Durham, D., Boyle, J., Cohen, R., Herzog, S., Rajan, R., and Sastry, A. (2000). The COPS (Common Open Policy Service) Protocol, IETF RFC 2748, January.

[12] ITU-T. Gateway Control Protocol: Version 2. (2002). Recommendation H.248, International Telecommunication Union, May.

[13] Schulzrinne, H., et al., RTP: A Transport Protocol for Real-Time Applications, (2003). IETF RFC 3550, Standard 64, July.

[14] Baugher, M., McGrew, D., Naslund, M., Carrara, E., and. Norrman, K. (2004) The Secure Realtime Transport Protocol (SRTP), IETF RFC 3711, March.

[15] 3GPP TR 22.934 version 8.0, release 8, (2008). Feasibility Study on 3GPP System to Wireless Local Area Network (WLAN) interworking.

[16] Zahirul.,I and Mirza, G. (2008). “A Comparative analysis on traditional Queuing and Hybrid Queuing Mecha nism of Vo IP's QoS Pro pert ies" International Journal Of Advance Innovations, Thoughts \& Idea, vol.2 (2), pp-19.

[17] Safdar, R., Asif, A., Saad, N. M., Nasrullah, A., and Mohd, Z. Y. (2011). "Tight Coupling Internetworking Between UMTS and WLAN: Challenges, Design Architectures and Simulation Analysis", International Journal of Computer Networks (IJCN), Volume (3) : Issue (2), pp-116-134. 